\title{
Sagan Dalya Tea, a New "Old" Probable Adaptogenic Drug: Metabolic Characterization and Bioactivity Potentials of Rhododendron adamsii Leaves
}

\author{
Daniil N. Olennikov ${ }^{1, * \mathbb{D}}$, Vyacheslav M. Nikolaev ${ }^{2}$ and Nadezhda K. Chirikova $^{3}$ \\ 1 Laboratory of Medical and Biological Research, Institute of General and Experimental Biology, \\ Siberian Division, Russian Academy of Science, 670047 Ulan-Ude, Russia \\ 2 Department of the Adaptation Mechanisms Study, Yakutsk Scientific Center of Complex Medical Problems, \\ 677000 Yakutsk, Russia; nikolaev1126@mail.ru \\ 3 Department of Biology, Institute of Natural Sciences, North-Eastern Federal University, \\ 677027 Yakutsk, Russia; hofnung@mail.ru \\ * Correspondence: olennikovdn@mail.ru; Tel.: +7-902-160-06-27
}

check for updates

Citation: Olennikov, D.N.; Nikolaev, V.M.; Chirikova, N.K. Sagan Dalya Tea, a New “Old" Probable

Adaptogenic Drug: Metabolic

Characterization and Bioactivity Potentials of Rhododendron adamsii Leaves. Antioxidants 2021, 10, 863. https://doi.org/10.3390/

antiox10060863

Academic Editor: Evangelos Zoidis

Received: 16 May 2021

Accepted: 26 May 2021

Published: 27 May 2021

Publisher's Note: MDPI stays neutral with regard to jurisdictional claims in published maps and institutional affiliations.

Copyright: (C) 2021 by the authors. Licensee MDPI, Basel, Switzerland. This article is an open access article distributed under the terms and conditions of the Creative Commons Attribution (CC BY) license (https:/ / creativecommons.org/licenses/by/ $4.0 /)$.

\begin{abstract}
Adams' rhododendron (Rhododendron adamsii Rehder) or Sagan Dalya tea is a famous Siberian evergreen medical plant of the Ericaceae family used in traditional medicines of Buryats, Yakuts, and Mongols as a tonic, stimulant, and adaptogenic drug. The high popularity of R. adamsii coupled with poor scientific knowledge prompted the addressing of gaps related to metabolic and biomedical data of Sagan Dalya tea. The application of solid-phase extraction and liquid chromatography-mass spectrometric techniques for the metabolomic study of $R$. adamsii leaf extracts resulted in the identification of more than 170 compounds, including carbohydrates, organic acids, simple phenol glycosides, triterpene glycosides, flavonoids, prenylated phenols, benzoic acid derivatives, hydroxycinnamates, dihydrochalcones, catechins, and procyanidins, most of which were identified for the first time in the plant. Extended surveys of the seasonal content of all detected compounds prove that specific metabolite variations reflect the bioactivity of $R$. adamsii extracts. Regarding in vitro methods, the expressed antioxidant potential of $R$. adamsii extracts was investigated via radical-scavenging, nitric oxide scavenging, and ferrous (II) ion chelating assays. The animal-based swimming to exhaustion test demonstrates the stimulating influence of $R$. adamsii extract on physical performance and endurance, concluding that the drug could act as an adaptogen. Thus, Sagan Dalya tea (R. adamsii) has confirmed its "old" application as a tonic remedy and requires further precise study as a novel adaptogenic plant.
\end{abstract}

Keywords: Rhododendron adamsii; Sagan Dalya; liquid chromatography-mass spectrometry; seasonal variation; antioxidant activity; adaptogen; swimming to exhaustion test

\section{Introduction}

Modern changes to human life, as well as the harmful effects of the environment observed in our time, often lead to a sharp decrease in the body's adaptive capacity and functional reserves. Therefore, the study of the mechanisms underlying the adaptation process and the search for new drugs and ways to increase the body's functional reserves are among the main aims of modern biomedical sciences. To increase the body's resistance to adverse factors, drugs of various groups are used, the most universal of which are natural adaptogens, which increase the body's performance and transfer it into a state of nonspecific increased resistance [1]. The relative safety and breadth of the therapeutic action of these natural remedies make them especially valuable for increasing the performance of people in unusual climatic conditions with static and dynamic industrial overloads, professional athletes, and the elderly, including those suffering from chronic diseases, alongside increasing the performance and mental activity of all people [2]. The effects of adaptogens on the human body are multi-faceted: adaptogens exhibit immunostimulating 
activity and improve anabolism, stimulate the central nervous and endocrine systems, modulate the sensitivity of cell receptors to hormones and the selective permeability of biological membranes, regulate the expression of many genes, have an antioxidant effect, and activate energy enzymes exchange, which ultimately leads to the economization of the metabolism and adaptation of the body to the unfavorable environment [3]. Due to their unique properties, many natural adaptogens are widely used, such as ginseng (Panax ginseng C.A.Mey.), roseroots (Rhodiola rosea L., Sedum roseum (L.) Scop.), and devil's bush (Eleutherococcus senticosus (Rupr. \& Maxim.) Maxim.) [4], while other adaptogens are currently being comprehensively researched and implemented in pharmacological practice.

Globally, Rhododendron adamsii Rehder $\{R$. fragrans (Adams) Maxim., Azalea fragrans Adams\}, or Adams' rhododendron (Figure 1), is a lesser-known adaptogen species, however, it is widely used in Siberia. Rhododendron adamsii is a small, evergreen shrub of the Ericaceae family, with dense rusty branches and thick, leathery, matt green wintering leaves [5]; when the leaves of the plant are touched, a greasy aromatic wax that thickly covers the leaves remains on the skin. Rhododendron adamsii grows in the mountains in the subalpine zone and, less commonly, in the alpine and upper forest zones of Central and Eastern Siberia and the Far East.



Figure 1. Rhododendron adamsii Rehder (Sagan Dalya) in its natural habitat (Republic Sakha Yakutia, Lena River delta, Kubalakh-Aryta Island, Orto-Khaya Mountain, tundra).

Rhododendron adamsii has numerous folk names, such as Sagan Dalya tea, White Wing, or Belgorod tea, owing to the story that the mountain spirit lives in it and helps to recover the health of warriors. The history of the Sagan Dalya name is enveloped in many poetic legends; according to one such legend, two lovers, Sagan and Dalya were separated by the evil shamaness, causing tears of the girl to fall to the ground and turn into the evergreen flowering shrubs also known as White Wing or Sagan Dalya [6]. In Buddhist mythology, Sagan Dalya is one of the seven plants surrounding the teacher of healing, the All-Enlightened Bhaishajyaguru [7].

Ethnopharmacological data indicates that the Buryat medicinal decoction of R. adamsii leaves (саган дали) is used as an elixir to strengthen the human organism [8]. Yakutian nomads used $R$. adamsii (хаскарa) decoction as a stimulant, diaphoretic, antipyretic, antibacterial, and analgesic drug [9]. In Mongolia, Buryatia, and Altai, shamans traditionally drink $R$. adamsii decoction as a tonic beverage, to enter a trance, and as a panacea for any disease. In Tibet, $R$. adamsii decoction is used to treat nervous disorders while, throughout Siberia, it is known as a powerful energy drink [10]. 
Current scientific information concerning $R$. adamsii metabolites remains insufficient, despite public interest and the wide use of this plant. Siberian samples of $R$. adamsii leaves and stems have been studied via GC/MS to elucidate their essential oil compositions, with nerolidol (9-29\%), $\beta$-farnesene (9-35\%), 4-phenyl-2-butanone (3-12\%), and aromadendrene (3-10\%) found to be the dominant compounds [11]. Some flavonoids have been characterized in ethanol extracts of R. adamsii leaves and stems, including myricetin, quercetin, dihydroquercetin, and rutin [12]. A recent study of the $\mathrm{CO}_{2}$ extract of $R$. adamsii leaves and branches showed a predomination of lipophilic components, including fatty acids, sterols, triterpenes, and some phenolic aglycones (flavonoids, coumarins) [13]. Additionally, at present, no data exists regarding the methods of application and the biological activity of $R$. adamsii, which has led to the emergence of many legends, as well as outright speculation, concerning the effectiveness of this plant.

As part of the ongoing work involving the metabolomic study of Siberian rhododendrons [14-16], we detail the first analysis of methanolic extracts of R. adamsii extracts in relation to seasonal metabolite variation using HPLC-PDA-ESI-tQ-MS (high-performance liquid chromatography with photodiode array detection and electrospray ionization triple quadrupole mass spectrometric detection) techniques. For the first time, the antioxidant and adaptogenic potentials of $R$. adamsii extracts were studied, demonstrating high effectiveness.

\section{Materials and Methods}

\subsection{Plant Material and Chemicals}

All samples of Rhododendron adamsii (215 samples totally) were collected in the tundra habitat in Republic Sakha Yakutia, Lena River delta, Kubalakh-Aryta Island, Orto-Khaya Mountain $\left(72^{\circ} 26^{\prime} 22.0^{\prime \prime} \mathrm{N}, 126^{\circ} 18^{\prime} 09.0^{\prime \prime}\right.$ E, $280 \mathrm{~m}$ a.s.1.) in January (15.I.2019-17.I.2019; 15 samples), March (12.III.2019-15.III.2019; 19 samples), May (16.V.2019-20.V.2019; 26 samples), June (12.VI.2019-16.VI.2019; 31 samples), July (15.VII.2019-17.VII.2019; 42 samples), August (17.VIII.2019-22.VIII.2019; 36 samples), October (12.X.2019-15.X.2019; 27 samples), and December (19.XII.2019-23.XII.2019; 19 samples). One sample consisted of 10-20 leaves (average length $2 \mathrm{~cm}$, height $1 \mathrm{~cm}$ ) collected from one bush. The species was authenticated by Dr. N.I. Kashchenko (IGEB SB RAS, Ulan-Ude, Russia). The plant material was dried in the ventilated heat oven at $40{ }^{\circ} \mathrm{C}$ within $7-10$ days and stored at $3-4{ }^{\circ} \mathrm{C}$ before analysis. The reference compounds were purchased from Cayman Chemicals (Ann Arbor, MI, USA), ChemFaces (Wuhan, Hubei, China), Extrasynthese (Lyon, France), MCE Med Chem Express (Monmouth, NJ, USA), Sigma-Aldrich (St. Louis, MO, USA), and Wuhan Chem Norm Biotech Co., Ltd. (Wuhan, China) (Table S1).

\subsection{Plant Extracts Preparation}

The extracts of R. adamsii leaves for the general chemical composition and preliminary bioactivity study were prepared from the powdered plant samples (50-100 g) extracted by appropriate solvent (water, methanol 20-100\%) with sonication (40 $\mathrm{min}, 40^{\circ} \mathrm{C}$, ultrasound power $100 \mathrm{~W}$, frequency $35 \mathrm{kHz}$ ). The chilled $\left(20^{\circ} \mathrm{C}\right.$ ) liquid was consequently filtered (filter paper), concentrated in vacuo until dryness, milled, and stored at $4{ }^{\circ} \mathrm{C}$ before analysis. The seasonal variation of metabolites and bioactivity of $R$. adamsii leaves analyzed for the extracts obtained by using $40 \%$ methanol as a solvent in the same extraction conditions.

\subsection{Chemical Composition Analysis}

UV-Vis spectrophotometer SF-200 (OKB Spectr, Saint Petersburg, Russia) was used for spectrophotometric quantitative determination of total protein (as $\mathrm{mg} / \mathrm{g}$ BSA equivalents) [17], total soluble carbohydrates (as $\mathrm{mg} / \mathrm{g}$ glucose equivalents) [18], polysaccharides (as mg/g glucose equivalents) [19], phenolics (as $\mathrm{mg} / \mathrm{g}$ gallic acid equivalents) [20], flavonols (as $\mathrm{mg} / \mathrm{g}$ hyperoside equivalents) [21], flavanols (as $\mathrm{mg} / \mathrm{g}$ taxifolin equivalents) [22], catechins (as mg/g (+)-catechin equivalents) [23], and procyanidins (as $\mathrm{mg} / \mathrm{g}$ 
procyanidin $B_{1}$ equivalents) [24] in R. adamsii extracts. All the analyses were carried out five times and the data were expressed as mean value \pm standard deviation (S.D.).

\subsection{Antioxidant Activity}

Microplate spectrophotometric assays were used to study the scavenging activity of $R$. adamsii extracts against 2,2-diphenyl-1-picrylhydrazyl radicals (DPPH ${ }^{\circ}$ [25], 2,2'-azinobis(3-ethylbenzothiazoline-6-sulfonic acid) cation radicals $\left(\mathrm{ABTS}^{\bullet+}\right.$ ) [26], N,N-dimethyl-pphenylenediamine radicals $\left(\mathrm{DMPD}^{\bullet+}\right)$ [27], superoxide anion radicals $\left(\mathrm{O}_{2}{ }^{\bullet-}\right)$ [28], hydroxyl radicals $\left({ }^{\bullet} \mathrm{OH}\right)$ [28], and chloride radicals $\left(\mathrm{Cl}^{\bullet}\right)$ [29]. Carotene bleaching spectrophotometric assay used $\beta$-carotene as a substrate (Sigma-Aldrich, cat. No. C9750) [30] and nitric oxide (II) scavenging assay used sodium nitroprusside as NO source [31]. Ferrous (II) ion chelating activity was studied by spectrophotometric assay [32]. Trolox (cat. No. 238813, $\geq 97 \%$; Sigma-Aldrich) was used as a reference standard $(1-100 \mu \mathrm{g} / \mathrm{mL}$ in methanol). All the analyses were carried out five times and the data were expressed as mean value \pm standard deviation (S.D.).

\subsection{Adaptogenic Activity}

\subsubsection{One-Step Swimming to Exhaustion Test}

The mice $(n=90)$ were randomly divided into ten groups received saline $(0.9 \% \mathrm{NaCl}$; $0.5 \mathrm{~mL}), R$. adamsii leave extract obtained by the various solvent $(0-100 \%$ methanol) at dose $50 \mathrm{mg} / \mathrm{kg}$, and Rodiola rosea rhizome extract ( $5 \%$ rosavins, Vitaforest Ltd., Saint Petersburg, Russia; $50 \mathrm{mg} / \mathrm{kg}$ ). In $60 \mathrm{~min}$, the mice were individually placed in a glass cylinder (height $40 \mathrm{~cm}$, diameter $20 \mathrm{~cm}$ ) filled with water (height $15 \mathrm{~cm} ; 22 \pm 1{ }^{\circ} \mathrm{C}$ ) and exhaustive swimming of rodents continued until the first immersion in the water. After the swimming sessions, the mice were towel-dried and returned to their housing. Each animal was used only once. The swimming time was measured using a stopwatch and was expressed in min. The experiment was realized in two versions, the first was the one-day application of plant extracts and the second was the 10-day application of plant extracts.

\subsubsection{Two-Step Swimming to Exhaustion Test}

The mice $(n=54)$ were randomly divided into six groups received saline $(0.9 \% \mathrm{NaCl}$; $0.5 \mathrm{~mL}$ ), R. adamsii leave extract (January, May, July, October samples) at dose $50 \mathrm{mg} / \mathrm{kg}$, and $R$. rosea extract $(50 \mathrm{mg} / \mathrm{kg}$ ) during 10 days and on the 10th day, the rodents were tested as described in Section 2.5.1. One hour later, the mice have been retested in the same conditions. The swimming times at each step were measured using a stopwatch and were expressed in min. At the end of the experiment, laboratory animals were decapitated and the homogenates of skeletal muscles (quadriceps femoris) and liver, and blood serum were assayed for the following biochemical parameters: skeletal muscles-adenosine triphosphate (fluorimetric ATP assay kit; Sigma-Aldrich, cat. No. MAK190), creatine phosphate (colorimetric phosphocreatine PCr ELISA kit; Abbexa Ltd., Cambridge, UK, cat. No. abx258965) and lactate (fluorimetric lactate assay kit; Sigma-Aldrich, cat. No. MAK064); blood serum — pyruvic acid (fluorimetric pyruvate assay kit; Sigma-Aldrich, cat. No. MAK071), glucose (colorimetric glucose assay kit; Sigma-Aldrich, cat. No. MAK264), malondialdehyde (colorimetric MDA assay kit; Abcam, Cambridge, UK, cat. No. ab238537), and catalase (catalase colorimetric activity kit; Invitrogen, Carlsbad, CA, USA, cat. No. EIACATC); liver-glycogen (colorimetric glycogen assay kit; Cayman Chemical, Ann Arbor, MI, USA, cat. No. 700480).

\subsection{Polyamide Solid-Phase Extraction (SPE)}

The pre-chromatographic solid-phase extraction (SPE) of R. adamsii extract was used before HPLC separation as described before [33,34] with a slight modification. Polyamide cartridges Chromabond (Polyamide 6; $6 \mathrm{~mL}, 1000 \mathrm{mg}$; Sorbent Technologies, Inc., Norcross, GA, USA) were eluted with methanol $(70 \mathrm{~mL})$ and water $(90 \mathrm{~mL})$. The extract of $R$. adamsii $(100 \mathrm{mg}$ ) was dissolved in $90 \%$ methanol $(1 \mathrm{~mL})$ followed by dilution of $20 \%$ methanol 
$(8 \mathrm{~mL})$, then centrifuged $(6000 \times g, 15 \mathrm{~min})$, and the supernatant was transferred in the volumetric flask $(10 \mathrm{~mL})$. Aliquots of internal standards were added to volumetric flask including 20-hydroxyecdysone $(100 \mu \mathrm{L} ; 500 \mu \mathrm{g} / \mathrm{mL}$ in $50 \%$ methanol; internal standardI), apigenin-7-O-glucoside $(150 \mu \mathrm{L} ; 400 \mu \mathrm{g} / \mathrm{mL}$ in $70 \%$ methanol; internal standard-II), apigenin-7-O-glucuronide $(150 \mu \mathrm{L} ; 400 \mu \mathrm{g} / \mathrm{mL}$ in $70 \%$ methanol; internal standard-III), and epigallocatechin 3-O-gallate $(100 \mu \mathrm{L} ; 1000 \mu \mathrm{g} / \mathrm{mL}$ in $30 \%$ methanol; internal standard-IV). The final volume reached $10 \mathrm{~mL}$ with $25 \%$ methanol in the volumetric flask (solution A). Solution A ( $2 \mathrm{~mL})$ was passed through polyamide SPE-cartridge and eluted with water (40 mL; eluate I), methanol (50 mL; eluate II), $0.55 \% \mathrm{NH}_{3}$ in methanol (50 mL; eluate III), pure water $(120 \mathrm{~mL})$, and DMSO $\left(40 \mathrm{~mL}\right.$; eluate IV). Eluates I-IV stored at $4{ }^{\circ} \mathrm{C}$ before chromatographic analysis (Section 2.7).

2.7. High-Performance Liquid Chromatography with Photodiode Array Detection and Electrospray Ionization Triple Quadrupole Mass Spectrometric Detection (HPLC-PDA-ESI-tQ-MS)

Qualitative chromatographic analysis of metabolic profiles of G. bifida extracts was done by high-performance liquid chromatography with photodiode array detection and electrospray ionization triple quadrupole mass spectrometric detection (HPLC-PDA-ESI-tQMS) technique using a liquid chromatograph LC-20 Prominence coupled with photodiode array detector SPD-M30A (wavelength range 200-600 nm), and triple-quadrupole mass spectrometer LCMS 8050 (all Shimadzu, Columbia, MD, USA) and C18 columns. Twoeluent gradient elution was used for the successful separation of compounds in four chromatographic modes (HPLC conditions) described in Table 1.

Table 1. HPLC conditions used for metabolite separation.

\begin{tabular}{|c|c|c|c|c|c|}
\hline $\begin{array}{c}\text { Mode No, } \\
\text { SPE Eluate (SPE } \\
\text { Eluent) }\end{array}$ & Column & $\begin{array}{c}{ }^{\circ} \mathrm{C} \\
\text { Column Temp., }\end{array}$ & $\begin{array}{l}\text { Eluents A/B } \\
\text { Composition }\end{array}$ & $\begin{array}{c}\text { Gradient Program, } \\
\% B\end{array}$ & $\begin{array}{c}\text { Flow Rate, } \\
\mu \mathrm{L} / \mathrm{min}\end{array}$ \\
\hline $\begin{array}{l}\text { Mode 1, SPE-1 } \\
\qquad\left(\mathrm{H}_{2} \mathrm{O}\right)\end{array}$ & $\begin{array}{c}\text { ProteCol }{ }^{\mathrm{TM}} \text { C18 } \\
\text { HPH125 (4.6 × } 250 \\
\text { mm, } 5 \mu \mathrm{m} \text {; Trajan } \\
\text { Scientific Australia } \\
\text { Pty Ltd., Ringwood, } \\
\text { Victoria, Australia) }\end{array}$ & 22 & $\begin{array}{c}0.2 \% \mathrm{HCOOH} \text { in } \\
\text { water } / \mathrm{MeCN}\end{array}$ & $\begin{array}{c}0-1 \min 5-6 \%, 2-5 \min \\
6-8 \%, 5-8 \min 8-15 \% \\
8-15 \min 15-29 \% \\
15-20 \min 29-5 \% \text { B }\end{array}$ & 100 \\
\hline $\begin{array}{l}\text { Mode 2, SPE-2 } \\
\quad(\mathrm{MeOH})\end{array}$ & $\begin{array}{c}\text { GLC Mastro }(2.1 \times \\
150 \mathrm{~mm}, 3 \mu \mathrm{m} ; \\
\text { Shimadzu, Kyoto, } \\
\text { Japan })\end{array}$ & 30 & $\begin{array}{c}0.5 \% \mathrm{HCOOH} \text { in } \\
\text { water } / 0.5 \% \\
\mathrm{HCOOH} \text { in } \mathrm{MeCN}\end{array}$ & $\begin{array}{c}0-2 \min 3-8 \%, 2-5 \min \\
8-9 \%, 5-12 \min 9-36 \% \\
12-13 \min 36-59 \% \\
13-15 \min 59-78 \% \\
15-22 \min 78-3 \%\end{array}$ & 150 \\
\hline $\begin{array}{l}\text { Mode } 3, \mathrm{SPE}-3 \\
\left(0.55 \% \mathrm{NH}_{3} \text { in }\right. \\
\mathrm{MeOH})\end{array}$ & $\begin{array}{c}\text { GLC Mastro }(2.1 \times \\
150 \mathrm{~mm}, 3 \mu \mathrm{m} ; \\
\text { Shimadzu, Kyoto, } \\
\text { Japan })\end{array}$ & 28 & $\begin{array}{c}0.5 \% \mathrm{HCOOH} \text { in } \\
\text { water } / 0.5 \% \\
\mathrm{HCOOH} \text { in } \mathrm{MeOH}\end{array}$ & $\begin{array}{c}0-1 \mathrm{~min} 5-12 \%, 1-3 \\
\min 12-16 \%, 3-7 \mathrm{~min} \\
16-29 \%, 7-11 \mathrm{~min} \\
29-49 \%, 11-15 \mathrm{~min} \\
49-87 \%, 15-25 \mathrm{~min} \\
87-5 \%\end{array}$ & 150 \\
\hline $\begin{array}{l}\text { Mode 4, SPE-4 } \\
\text { (DMSO) }\end{array}$ & $\begin{array}{c}\text { Acclaim } 120 \text { C18 (2.1 } \\
\times 150 \text { mm, } 2.2 \mu \mathrm{m} ; \\
\text { Dionex, Sunnyvale, } \\
\text { CA, USA) }\end{array}$ & 25 & $\begin{array}{c}0.1 \% \text { TFA in } \\
\text { water } / 0.1 \% \text { TFA in } \\
\text { MeCN }\end{array}$ & $\begin{array}{c}0-3 \min \\
0-5 \%, 3-8 \min 5-10 \% \\
8-15 \min 8-14 \%, 15-17 \\
\min 14-33 \%, 17-22 \\
\min 33-59 \%, 22-30 \\
\min 59-73 \%, 30-40 \\
\min 73-0 \%\end{array}$ & 300 \\
\hline
\end{tabular}

The injection volume was $1 \mu \mathrm{L}$ in all modes. The UV-Vis spectra were registered in the spectral range of 200-600 nm. Mass spectrometric detection was performed in negative ESI mode and the temperature levels of ESI interface, desolvation line, and heat 
block were $300{ }^{\circ} \mathrm{C}, 250{ }^{\circ} \mathrm{C}$, and $400{ }^{\circ} \mathrm{C}$, respectively, and the flow of nebulizing gas $\left(\mathrm{N}_{2}\right)$, heating gas (air), and collision-induced dissociation gas (Ar) were $3 \mathrm{~L} / \mathrm{min}, 10 \mathrm{~L} / \mathrm{min}$, and $0.3 \mathrm{~mL} / \mathrm{min}$, respectively. The mass spectra were registered as $3 \mathrm{kV}$ source voltage and collision energy $-10-35 \mathrm{eV}$ by the scanning range of $m / z$ 80-2000. The managing of the LC-MS system was realized by LabSolution's workstation software equipped with the inner LC-MS library. The final identification of metabolites performed after an integrated analysis of retention time, ultraviolet, and mass spectra in comparison with the reference standards and literature data.

\subsection{Metabolite Quantification}

The quantitative analysis of compounds found in R. adamsii was done using the above described HPLC-PDA-ESI-tQ-MS conditions (Section 2.7) and full scan MS peak area used for calculation. One hundred and one compounds were quantitatively analyzed using 55 reference standards (Table S1). Each compound was carefully weighed (10 mg), dissolved in the methanol-DMSO mixture (1:1) in volumetric flasks $(10 \mathrm{~mL})$, and the reference standard calibration curves were built using the stock solutions in methanol (1-100 $\mu \mathrm{g} / \mathrm{mL}$ ). Mass spectrometric peak area data were used to plot 'concentration-peak area' graphs and determination the validation criteria (correlation coefficients, $r^{2}$; standard deviation, $S_{Y X}$; limits of detection, LOD; limits of quantification, LOQ; and linear ranges) calculated as described previously [35] (Table S2). All quantitative analyses were carried out five times, and the data were expressed as mean value \pm standard deviation (S.D.).

\subsection{HPLC-UV Assay Coupled with DPPH Precolumn Incubation}

The extract of $R$. adamsii (July sample, $100 \mathrm{mg}$ ) was sonically dissolved in $40 \%$ methanol (5 mL), centrifuged $(6000 \times g, 20 \mathrm{~min})$, and used for HPLC-PAD separation without SPE pretreatment. The aliquote of $R$. adamsii extract solution $(50 \mu \mathrm{L})$ was mixed with $0.5 \%$ $\mathrm{DPPH}^{\bullet}$ radicals solution in methanol $(50 \mu \mathrm{L})$ and incubated $15 \mathrm{~min}$ at $20^{\circ} \mathrm{C}$. The probe without $\mathrm{DPPH}^{\bullet}$ radicals preincubation was diluted with methanol (1:1) before separation. High-performance liquid chromatography with UV detection coupled with $\mathrm{DPPH}^{\bullet}$ radicals preincubation performed by microcolumn liquid chromatography MiliChrom A-02 (EcoNova, Novosibirsk, Russia). The apparatus was coupled with UV detector 190-360 EcoNova (EcoNova, Novosibirsk, Russia) and ProntoSIL-120-5-C18 AQ column $\left(50 \times 1 \mathrm{~mm}, 1 \mu \mathrm{m}\right.$; Metrohm AG, Herisau, Switzerland) with column temperature of $30^{\circ} \mathrm{C}$. The eluent composition was $0.2 \mathrm{M} \mathrm{LiClO}_{4}$ in $0.01 \mathrm{M} \mathrm{HClO}_{4}$ for eluent $\mathrm{A}$ and $0.01 \mathrm{M} \mathrm{HClO}_{4}$ in $\mathrm{MeCN}$ for eluent $\mathrm{B}$. The injection volume was $1 \mu \mathrm{L}$, and the elution flow was $150 \mu \mathrm{L} / \mathrm{min}$. The gradient separation was used programmed as $0.0-26.6 \mathrm{~min} 5-100 \% \mathrm{~B}, 26.6-28.6 \mathrm{~min}$ $100 \% \mathrm{~B}$. The chromatograms were recorded at $270 \mathrm{~nm}$. Finally, the chromatograms of untreated and $\mathrm{DPPH}^{\bullet}$ radicals probes were overlapped and compared. The reduction of chromatographic peak area indicating the radical-scavenging potential of compounds eluted in corresponding peaks.

\subsection{Statistical and Multivariate Analysis}

Statistical analyses were performed by one-way analysis of variance, and the significance of the mean difference was determined by Duncan's multiple range test. Differences at $p<0.05$ were considered statistically significant. The results are presented as mean values \pm standard deviations (S.D.) of some replicates. The linear regression analysis and generation of calibration graphs were conducted using Advanced Grapher 2.2 (Alentum Software Inc., Ramat-Gan, Israel). Principal component analysis based on a data matrix (171 markers $\times 215$ samples) was performed using Graphs 2.0 utility for Microsoft Excel (Komi NTc URO RAN, Syktyvkar, Russia) to generate an overview for group clustering. 


\section{Results and Discussion}

\subsection{Chemical Composition and Bioactivity of Rhododendron Adamsii Leaves: Impact of} Solvent Type

Prior to the in-depth chemical and biological study of $R$. adamsii leaves, we analyzed various extracts to identify the best solvent for providing the most active remedy. Various methanols (0-100\%) were used and characterized chemically (Table 2). The total yield of the extracts varied from $12.5 \%$ to $35.0 \%$ of dry plant weight. The basic nutrient content of $R$. adamsii leaves fluctuated depending on the methanol concentration: proteins and soluble carbohydrates showed the highest amounts in water extractions $(23.69 \mathrm{mg} / \mathrm{g}$ and $326.03 \mathrm{mg} / \mathrm{g}$, respectively), while lipids dominated in the pure methanol extract $(254.12 \mathrm{mg} / \mathrm{g})$. The main explanation for the distribution of nutrients in extracts lies in the rule 'like solves like', i.e., hydrophilic solvents extract hydrophilic compounds, and vice versa; the same rule applies to polysaccharides due to their high content in the water extractions $(57.60 \mathrm{mg} / \mathrm{g})$.

Table 2. Chemical composition and DPPH${ }^{\bullet}$ scavenging activity of $R$. adamsii leave extracts, \pm S.D.

\begin{tabular}{|c|c|c|c|c|c|c|c|c|}
\hline \multirow{2}{*}{ Parameter } & \multicolumn{8}{|c|}{ Solvent Type, \% Methanol } \\
\hline & 0 & 20 & 40 & 50 & 60 & 70 & 80 & 100 \\
\hline Yield, g/100 g & $12.5 \pm 0.6$ & $17.5 \pm 0.8$ & $18.5 \pm 0.9$ & $27.9 \pm 1.1$ & $30.5 \pm 1.5$ & $35.0 \pm 1.7$ & $32.5 \pm 1.6$ & $32.0 \pm 1.4$ \\
\hline Protein, mg/g & $23.69 \pm 0.71$ & $11.27 \pm 0.31$ & $2.09 \pm 0.06$ & n.d. & n.d. & n.d. & n.d. & n.d. \\
\hline Lipids, mg/g & $1.18 \pm 0.04$ & $10.86 \pm 0.34$ & $53.69 \pm 1.61$ & $73.14 \pm 2.19$ & $86.03 \pm 2.44$ & $183.22 \pm 5.49$ & $229.17 \pm 6.87$ & $\begin{array}{c}254.12 \pm \\
12.70\end{array}$ \\
\hline $\begin{array}{c}\text { Soluble } \\
\text { carbohydrates, } \\
\mathrm{mg} / \mathrm{g}\end{array}$ & $326.03 \pm 8.15$ & $308.02 \pm 7.73$ & $153.62 \pm 3.94$ & $92.14 \pm 2.25$ & $76.02 \pm 1.83$ & $43.25 \pm 1.08$ & $20.63 \pm 0.51$ & $9.35 \pm 0.23$ \\
\hline $\begin{array}{l}\text { Polysaccharides, } \\
\mathrm{mg} / \mathrm{g}\end{array}$ & $57.60 \pm 1.73$ & $24.18 \pm 0.70$ & $3.02 \pm 0.06$ & n.d. & n.d. & n.d. & n.d. & n.d. \\
\hline $\begin{array}{l}\text { Phenolics, } \\
\mathrm{mg} / \mathrm{g}\end{array}$ & $163.15 \pm 0.48$ & $185.69 \pm 5.57$ & $293.35 \pm 8.79$ & $253.8 \pm 6.32$ & $197.54 \pm 5.82$ & $143.20 \pm 4.25$ & $127.03 \pm 3.80$ & $\begin{array}{c}108.27 \pm \\
3.20\end{array}$ \\
\hline $\begin{array}{c}\text { Flavonols, } \\
\mathrm{mg} / \mathrm{g}\end{array}$ & $97.86 \pm 1.95$ & $107.75 \pm 2.37$ & $138.88 \pm 2.78$ & $102.03 \pm 2.50$ & $90.64 \pm 1.90$ & $82.16 \pm 1.64$ & $74.81 \pm 1.42$ & $\begin{array}{c}64.59 \pm \\
1.29\end{array}$ \\
\hline $\begin{array}{c}\text { Flavanols, } \\
\mathrm{mg} / \mathrm{g}\end{array}$ & $10.21 \pm 0.18$ & $12.59 \pm 0.25$ & $16.84 \pm 0.31$ & $16.09 \pm 0.27$ & $14.69 \pm 0.25$ & $10.03 \pm 0.18$ & $5.63 \pm 0.11$ & $2.29 \pm 0.04$ \\
\hline $\begin{array}{c}\text { Catechins, } \\
\mathrm{mg} / \mathrm{g}\end{array}$ & $40.10 \pm 1.00$ & $48.52 \pm 1.28$ & $89.27 \pm 2.73$ & $88.41 \pm 2.65$ & $82.16 \pm 2.46$ & $61.01 \pm 1.65$ & $58.44 \pm 1.43$ & $\begin{array}{c}55.20 \pm \\
1.21\end{array}$ \\
\hline $\begin{array}{c}\text { Procyanidins, } \\
\mathrm{mg} / \mathrm{g}\end{array}$ & $14.85 \pm 0.52$ & $24.32 \pm 0.87$ & $27.98 \pm 0.95$ & $26.92 \pm 0.79$ & $25.08 \pm 0.75$ & $15.41 \pm 0.57$ & $6.65 \pm 0.21$ & $3.49 \pm 0.12$ \\
\hline $\begin{array}{c}\mathrm{DPPH}^{\bullet}, \mathrm{IC}_{50}, \\
\mu \mathrm{g} / \mathrm{mL}\end{array}$ & $10.47 \pm 0.31$ & $7.33 \pm 0.21$ & $4.82 \pm 0.14$ & $4.93 \pm 0.15$ & $5.67 \pm 0.17$ & $14.22 \pm 0.42$ & $16.06 \pm 0.48$ & $\begin{array}{c}25.81 \pm \\
0.77\end{array}$ \\
\hline
\end{tabular}

n.d.- not detected.

The phenolics are a class of compounds with a medium polarity that demonstrate optimal extractability using $40 \%$ methanol, with yields of $293.35 \mathrm{mg} / \mathrm{g}$ for total phenolic content, $138.88 \mathrm{mg} / \mathrm{g}$ for total flavonols, $16.84 \mathrm{mg} / \mathrm{g}$ for flavanols, $89.27 \mathrm{mg} / \mathrm{g}$ for catechins, and $27.98 \mathrm{mg} / \mathrm{g}$ for procyanidins. This impacted the radical scavenging ability of $R$. adamsii extracts against 2,2-diphenyl-1-picrylhydrazyl radicals ( $\mathrm{DPPH}^{\bullet}$ ), well-known artificial free radicals used in the detection of plant antioxidants [36]. The $40 \%$ methanol extract demonstrated the best DPPH scavenging activity with $\mathrm{IC}_{50}$ value $4.82 \mu \mathrm{g} / \mathrm{mL}$ (half maximal inhibitory concentration). The use of solvents with lower or higher concentrations of methanol negatively affects the antioxidant potential of the extracts.

Early studies of rhododendrons showed good ability to accumulate phenolic compounds such as $R$. ponticum (349.53 mg/g) [37], R. pseudochrysanthum (319 mg/g), $R$. breviperulatum (265 mg/g), R. oldhamii $(264 \mathrm{mg} / \mathrm{g})$ [38], R. anthopogonoides (165 mg/g) [39], R. jasminiflorum $(48.11 \mathrm{mg} / \mathrm{g})$, R. konori $(41.17 \mathrm{mg} / \mathrm{g})$, and R. javanicum $(40.39 \mathrm{mg} / \mathrm{g})$ [40]. Flavonoids seem to be the major compound in R. ponticum $(311.16 \mathrm{mg} / \mathrm{g}$ ) [37], R. anthopogonoides (231.37 mg/g) [39], R. jasminiflorum $(8.92 \mathrm{mg} / \mathrm{g})$, R. javanicum $(6.15 \mathrm{mg} / \mathrm{g})$, and $R$. seranicum $(5.90 \mathrm{mg} / \mathrm{g}$ ) [40]. Information regarding total contents of the separated phenolic classes, such as flavonols, flavanols, catechins, and procyanidins, is not widely 
available from rhododendrons around the world. Despite this, the high antioxidant potential of rhododendrons is a well-known fact, as shown by the DPPH ${ }^{\bullet}$ values of $R$. ponticum ( $\mathrm{IC}_{50} 1.23 \mu \mathrm{g} / \mathrm{mL}$ ) [37], R. pseudochrysanthum, R. oldhamii ( $\mathrm{IC}_{50} 7.5 \mu \mathrm{g} / \mathrm{mL}$ ), $R$. kanehirai ( $\mathrm{IC}_{50} 7.7 \mu \mathrm{g} / \mathrm{mL}$ ) [38], R. przewalskii ( $\mathrm{IC}_{50} 31 \mu \mathrm{g} / \mathrm{mL}$ ) [41], R. anthopogonoides $\left(\mathrm{IC}_{50}\right.$ $63.75 \mu \mathrm{g} / \mathrm{mL})$ [39], R. jasminiflorum ( $29 \mu \mathrm{M}$ trolox/g), R. seranicum (28 $\mu \mathrm{M}$ trolox $/ \mathrm{g})$, and $R$. javanicum $(26 \mu \mathrm{M}$ trolox/g) [40] extracts.

Unlike the antioxidant properties of Rhododendron species, the impact of rhododendron extracts on physical endurance has not been studied previously, therefore, the adaptogenic potential of R. adamsii is disputed and requires further investigation; in our current study, we use the one-step swimming to exhaustion test of mice to examine this, using swimming time as an indicator of effectiveness. The extract of $R$. rosea roots (total rosavins $5 \%$ ) was used as a plant remedy known to enhance resistance activity to physical training [28]. The eight extracts of $R$. adamsii leaves created using $0-100 \%$ methanol as a solvent were studied using a single dose of $50 \mathrm{mg} / \mathrm{kg}$, the same dose as used for $R$. rosea extract (Figure 2). Preliminary experiments demonstrated lower $(25 \mathrm{mg} / \mathrm{kg})$ and higher doses $(100 \mathrm{mg} / \mathrm{kg})$ of the extracts to be less effective.

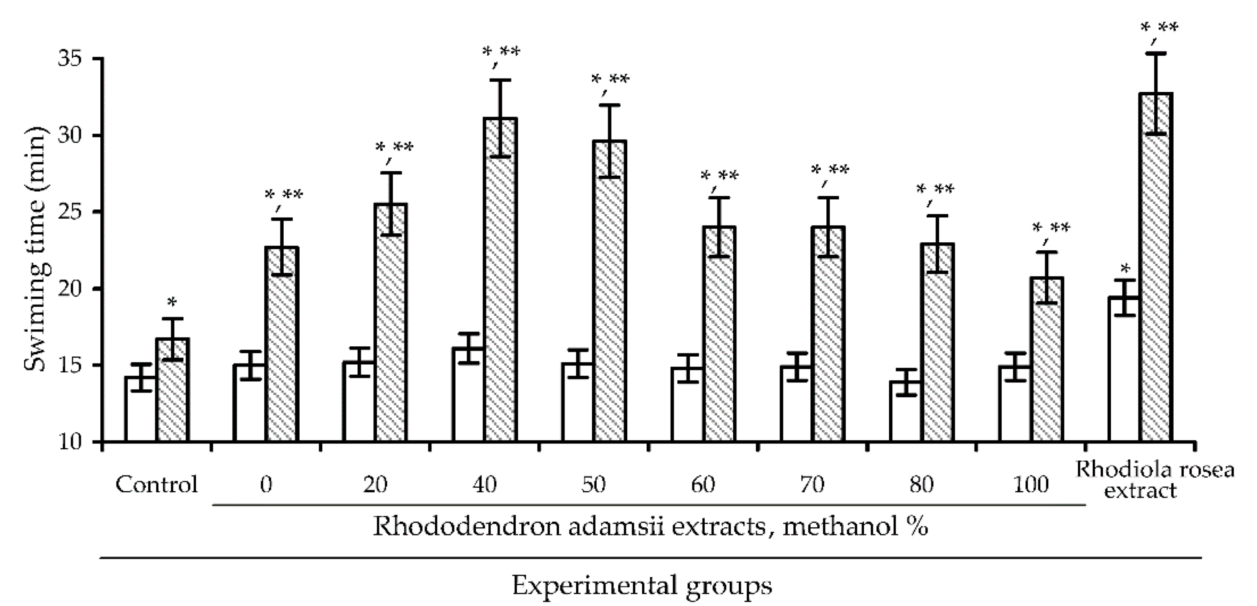

Figure 2. The effect of $R$. adamsii leaf extracts (solvent: $0-100 \%$ methanol) and $R$. rosea extract $(50 \mathrm{mg} / \mathrm{kg})$ on swimming time of mice in swimming to exhaustion test on day 1 (empty bars) and day 10 (shaded bars). ${ }^{*}-p<0.05$ vs. control group, day $1{ }^{* *}-p<0.05$ vs. control group, day 10 .

In the swimming test of mice experiments, one-day application of $R$. adamsii extract resulted in no statistically significant $(p<0.05)$ increase of swimming time of mice, in contrast to $R$. rosea extract, which resulted in a $36 \%$ increase in swimming time compared with the control group (19.4 min vs. $14.2 \mathrm{~min}$, respectively). The ten-day experiments demonstrated increases in animal endurance in all $R$. adamsii extract groups, with various levels of intensity. Of the extract groups, the $40 \%$ methanol extract showed the best effectiveness ( $31.1 \mathrm{~min}$ ) when compared to the $R$. rosea extract group (32.7 $\mathrm{min}$ ). Based on these results, we can conclude that $R$. adamsii extract enhances physical endurance, however, the properties of this endowment differ from those provided by $R$. rosea, as the latter medicine is characterized by a rapid influence on the animal subject while the $R$. adamsii extract's impact develops over time (cumulative effect). It is easily noted that the $40 \%$ methanol extract of $R$. adamsii leaves demonstrated the maximal antioxidant power and adaptogenic potential, indicating a possible linkage of both activities. Interestingly, a strong correlation between the radical scavenging ability of $R$. adamsii extracts against DPPH radicals and the swimming time of mice in swimming to exhaustion test on day 10 was observed with correlation coefficient $r=0.8118$ (Figure 3). Some researchers have previously expressed their views on the role of antioxidants in the realization of adaptogenic properties of plant extracts; these researchers have a cautious view that antioxidants decrease the risk of complications induced by oxidative stress, thus contributing to the positive effect of adaptogens [42,43]. In light of known data concerning stress-induced 
increases in free radical processes in mammals [1], it makes sense that a close relationship between adaptogens and antioxidant properties exists.

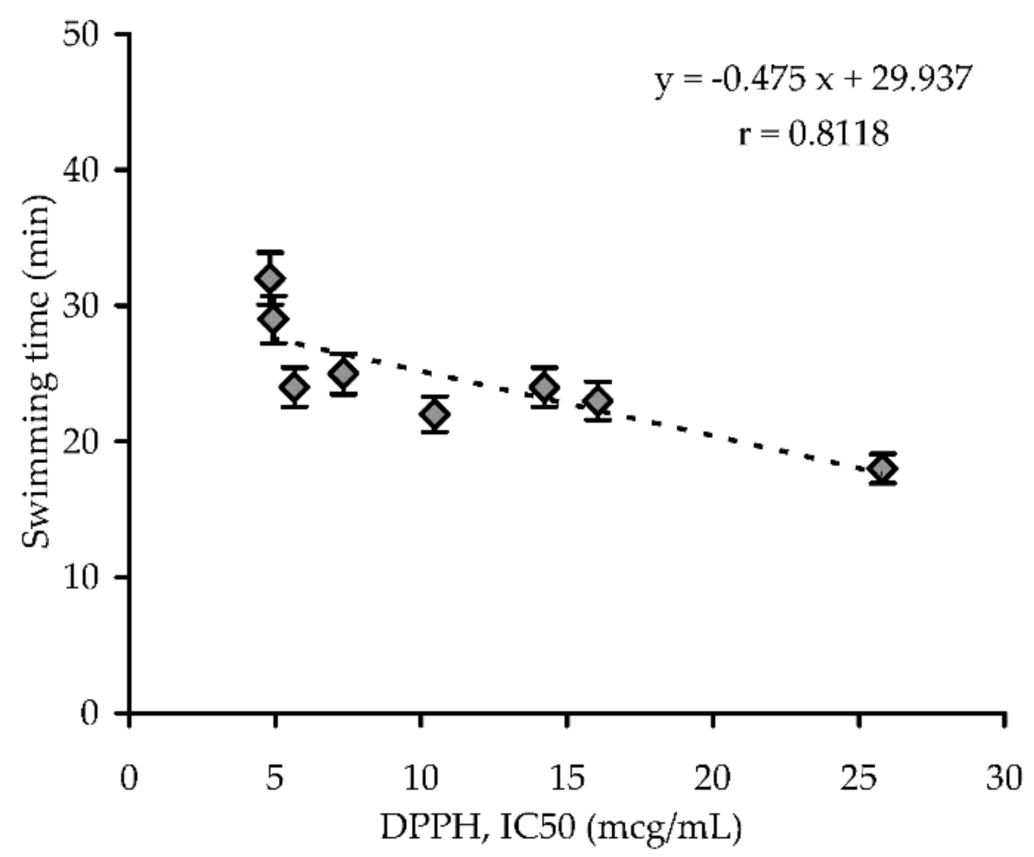

Figure 3. Correlation graph between radical scavenging ability of Rhododendron adamsii extracts against 2,2-diphenyl-1-picrylhydrazyl radicals (DPPH; independent variable $x$ in regression equation) and swimming time of mice in swimming to exhaustion test on day 10 (dependent variable $y$ in regression equation). $\mathrm{r}$-correlation coefficient.

\subsection{Rhododendron Adamsii Leaves Metabolites: LC-MS Characterisation and Seasonal Variation}

Rhododendron genus demonstrates the presence of numerous chemical classes with specific chromatographic behavior [9]. Critical to the success of metabolite separation are the preliminary procedures for purification and partitioning of the total plant extract on the specific fractions. Solid-phase extraction (SPE) is a helpful tool for the pre-treatment of plant samples prior to chromatographic analysis $[28,33,34]$. Application of polyamidebased SPE of methanol extract of $R$. adamsii leaves yielded four fractions enriched with highly hydrophilic compounds (SPE-1), neutral phenolics (SPE-2), acidic and acylated phenolics (SPE-3), and tannin-like compounds (SPE-4); this method avoided overlapping of the chromatographic zones and enabled better identification of the compounds.

To separate the majority of the methanol-extractable compounds of the R. adamsii leaves, we used high-performance liquid chromatography with photodiode array and electrospray ionization triple quadrupole mass spectrometric detection (HPLC-PDA-ESItQ-MS). Synchronous analysis of chromatographic mobility, ultraviolet spectra, and mass spectral pattern, and comparison of the data with reference standards or/and literature information $[12,13,26,28,28,33,35,44-55]$ resulted in the identification of 171 compounds (Figures 4 and 5, Table 3 and Table S3). Previously, only ten phenolics had been described in $R$. adamsii leaves, resulting in more than 160 originally documented here [11-13]. 




(a)



(b)

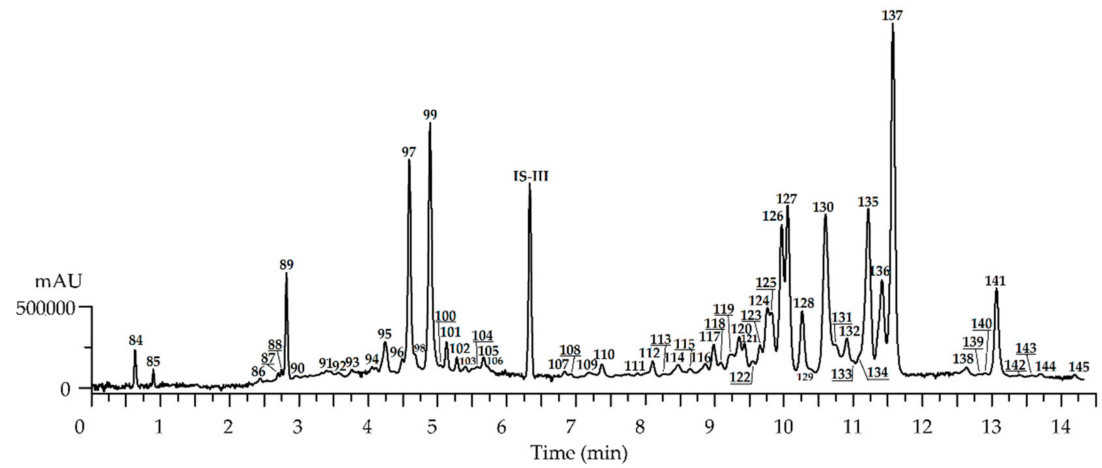

(c)

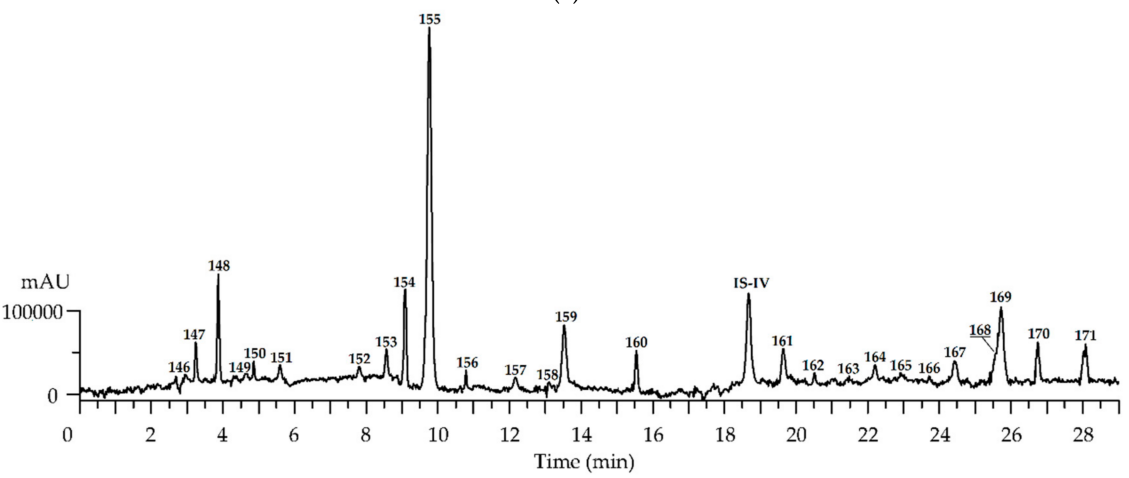

(d)

Figure 4. High-Performance Liquid Chromatography with Electrospray Ionization Triple Quadrupole Mass Spectrometric Detection (HPLC-ESI-tQ-MS) chromatogram (Total Ion Chromatogram (TIC) mode, negative ionization) of solid-phase extraction (SPE) eluates of $R$. adamsii leaves (July sample) extract: $\mathrm{H}_{2} \mathrm{O}$ eluate (SPE-1; a), $\mathrm{MeOH}$ eluate (SPE-2; b), $\mathrm{NH}_{3}-\mathrm{MeOH}$ eluate (SPE-3; c), DMSO eluate (SPE-4; d). Compounds are numbered as listed in Table 2. Internal standards used: 20hydroxyecdysone (IS-I), apigenin-7-O-glucoside (IS-II), apigenin-7-O-glucuronide (IS-III), epigallocatechin O-gallate (IS-IV). 


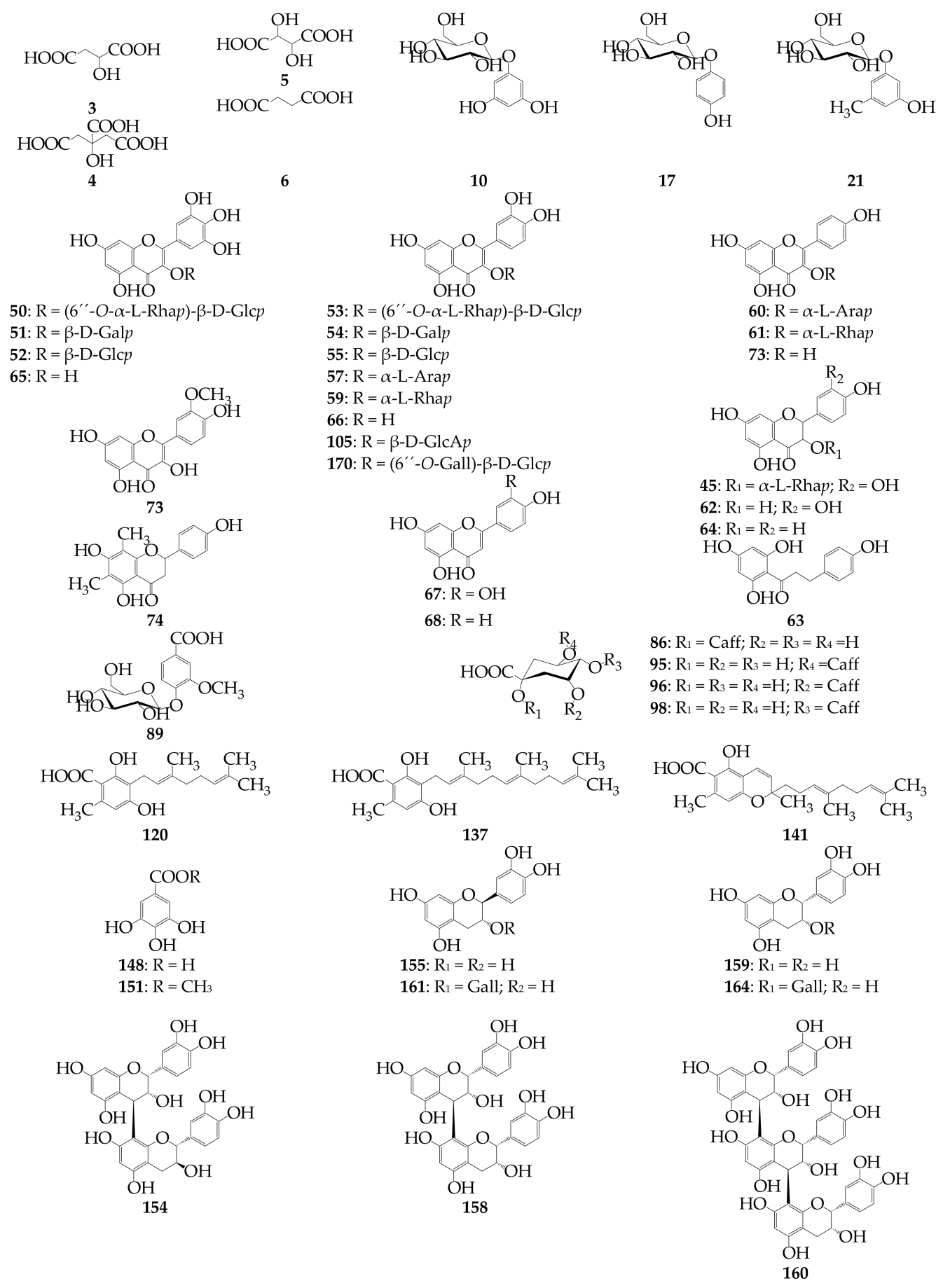

Figure 5. Structures of known compounds found in R. adamsii. Abbreviation used: Arap—arabinopyranose; Caff-Caffeoyl; Gall-Galloyl; Gal $p$-Galactopyranose; Glc $p$-Glucopyranose; GlcAp—Glucuronopyranose; Rhap —Rhamnopyranose. 
Table 3. Compounds 1-171 were found in R. adamsii leaves with their seasonal content.

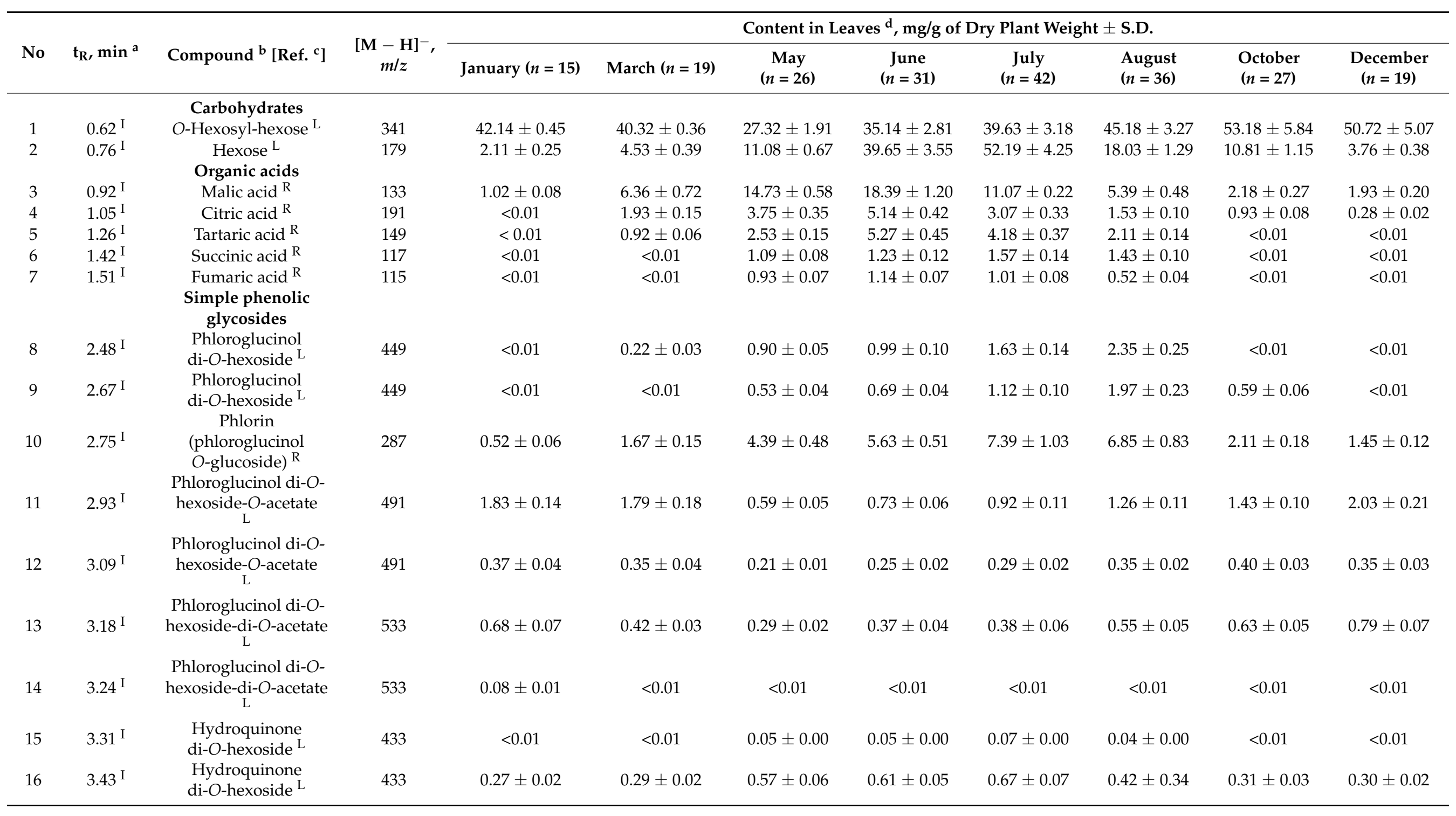


Table 3. Cont.

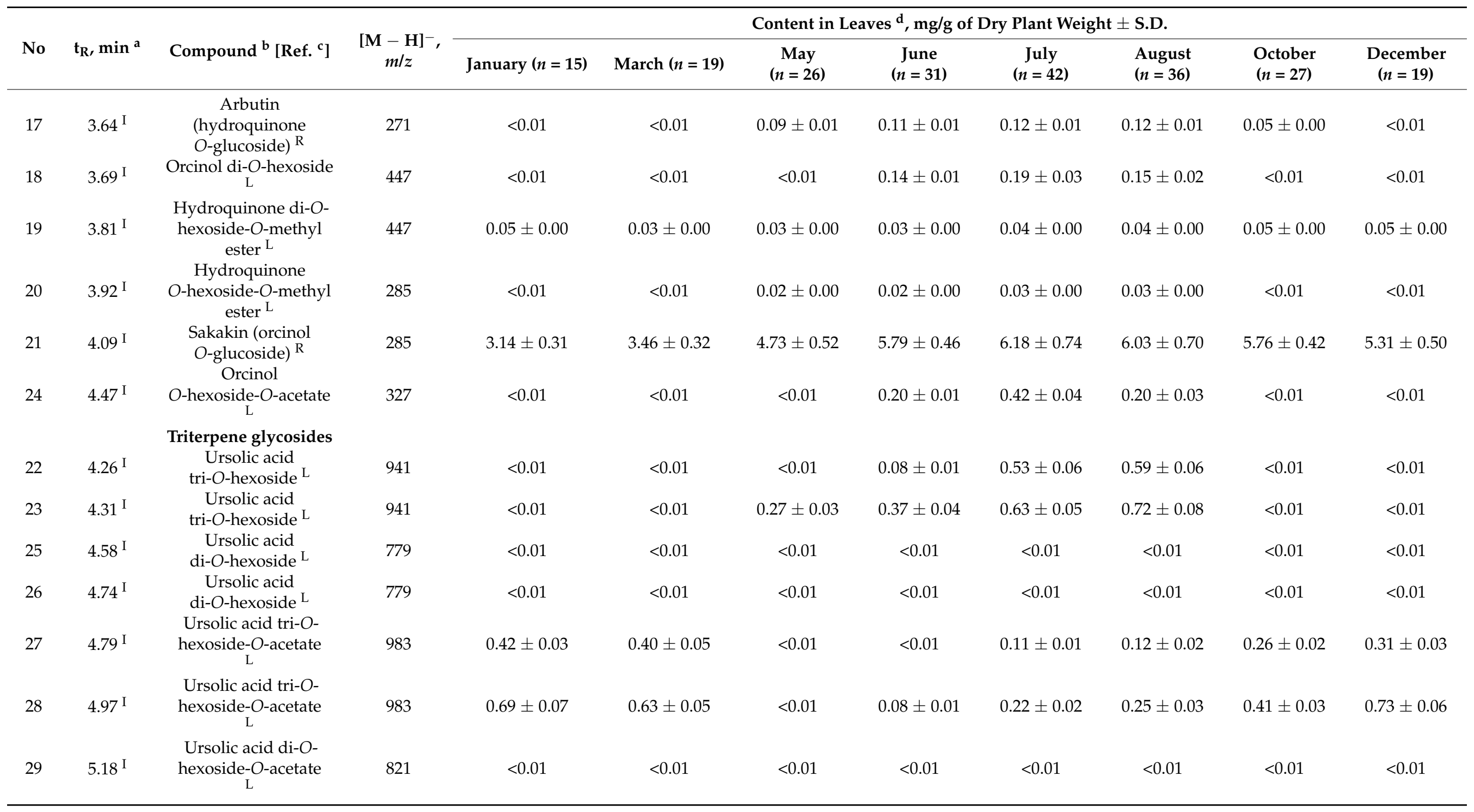


Table 3. Cont.

\begin{tabular}{|c|c|c|c|c|c|c|c|c|c|c|c|}
\hline \multirow[b]{2}{*}{ No } & \multirow[b]{2}{*}{$t_{R}, \min { }^{a}$} & \multirow[b]{2}{*}{ Compound $^{b}\left[\right.$ Ref. $\left.^{c}\right]$} & \multirow[b]{2}{*}{$\begin{array}{l}{[\mathbf{M}-\mathbf{H}]^{-}} \\
\quad m / z\end{array}$} & \multicolumn{8}{|c|}{ Content in Leaves $^{\mathrm{d}}, \mathrm{mg} / \mathrm{g}$ of Dry Plant Weight \pm S.D. } \\
\hline & & & & January $(n=15)$ & March $(n=19)$ & $\begin{array}{c}\text { May } \\
(n=26)\end{array}$ & $\underset{(n=31)}{\text { June }}$ & $\underset{(n=42)}{\text { July }}$ & $\begin{array}{l}\text { August } \\
(n=36)\end{array}$ & $\begin{array}{l}\text { October } \\
(n=27)\end{array}$ & $\begin{array}{c}\text { December } \\
(n=19)\end{array}$ \\
\hline 30 & $5.46^{\mathrm{I}}$ & $\begin{array}{l}\text { Ursolic acid } \\
\text { O-hexoside }{ }^{\mathrm{L}}\end{array}$ & 617 & $2.57 \pm 0.31$ & $4.85 \pm 0.32$ & $11.73 \pm 1.42$ & $15.37 \pm 1.50$ & $17.26 \pm 1.83$ & $17.54 \pm 1.85$ & $14.31 \pm 1.28$ & $3.06 \pm 0.36$ \\
\hline 31 & $5.53^{\mathrm{I}}$ & $\begin{array}{c}\text { Ursolic acid di-O- } \\
\text { hexoside-di-O-acetate } \\
\text { L }\end{array}$ & 863 & $<0.01$ & $<0.01$ & $<0.01$ & $<0.01$ & $<0.01$ & $<0.01$ & $<0.01$ & $<0.01$ \\
\hline 32 & $5.74^{\mathrm{I}}$ & $\begin{array}{c}\text { Ursolic acid di-O- } \\
\text { hexoside-di-O-acetate } \\
\text { L }\end{array}$ & 863 & $<0.01$ & $<0.01$ & $<0.01$ & $<0.01$ & $<0.01$ & $<0.01$ & $<0.01$ & $<0.01$ \\
\hline 33 & $7.87^{\mathrm{I}}$ & $\begin{array}{c}\text { Ursolic acid } \\
\text { O-hexoside-O-acetate } \\
\text { L }\end{array}$ & 659 & $<0.01$ & $<0.01$ & $<0.01$ & $<0.01$ & $<0.01$ & $<0.01$ & $<0.01$ & $<0.01$ \\
\hline 34 & $8.28^{\mathrm{I}}$ & $\begin{array}{c}\text { Ursolic acid } \\
\text { O-hexoside-O-acetate } \\
\text { L }\end{array}$ & 659 & $<0.01$ & $<0.01$ & $<0.01$ & $<0.01$ & $<0.01$ & $<0.01$ & $<0.01$ & $<0.01$ \\
\hline 35 & $10.26^{\mathrm{I}}$ & $\begin{array}{c}\text { Ursolic acid } O- \\
\text { hexoside-di-O-acetate } \\
\text { L }\end{array}$ & 701 & $<0.01$ & $<0.01$ & $<0.01$ & $<0.01$ & $<0.01$ & $<0.01$ & $<0.01$ & $<0.01$ \\
\hline 36 & $2.72^{\mathrm{II}}$ & $\begin{array}{c}\text { Flavonols } \\
\text { Glycosides: myricetin } \\
\text { derivatives } \\
\text { Myricetin } \\
\text { tri-O-hexoside-tri-O- } \\
\text { desoxyhexoside } \\
\text { L }\end{array}$ & 1241 & $<0.01$ & $<0.01$ & $<0.01$ & $<0.01$ & $<0.01$ & $<0.01$ & $<0.01$ & $<0.01$ \\
\hline 37 & $2.79 \mathrm{II}$ & $\begin{array}{c}\text { Myricetin } \\
\text { tri-O-hexoside-di-O- } \\
\text { desoxyhexoside } \\
\text { L }\end{array}$ & 1095 & $<0.01$ & $<0.01$ & $<0.01$ & $<0.01$ & $<0.01$ & $<0.01$ & $<0.01$ & $<0.01$ \\
\hline 46 & $4.09 \mathrm{II}$ & $\begin{array}{c}\text { Myricetin } \\
\text { di-O-hexoside-di-O- } \\
\text { desoxyhexoside } \\
\text { L }\end{array}$ & 933 & $<0.01$ & $<0.01$ & $1.53 \pm 0.11$ & $1.67 \pm 0.12$ & $1.99 \pm 0.17$ & $1.52 \pm 0.12$ & $0.35 \pm 0.04$ & $<0.01$ \\
\hline
\end{tabular}


Table 3. Cont.

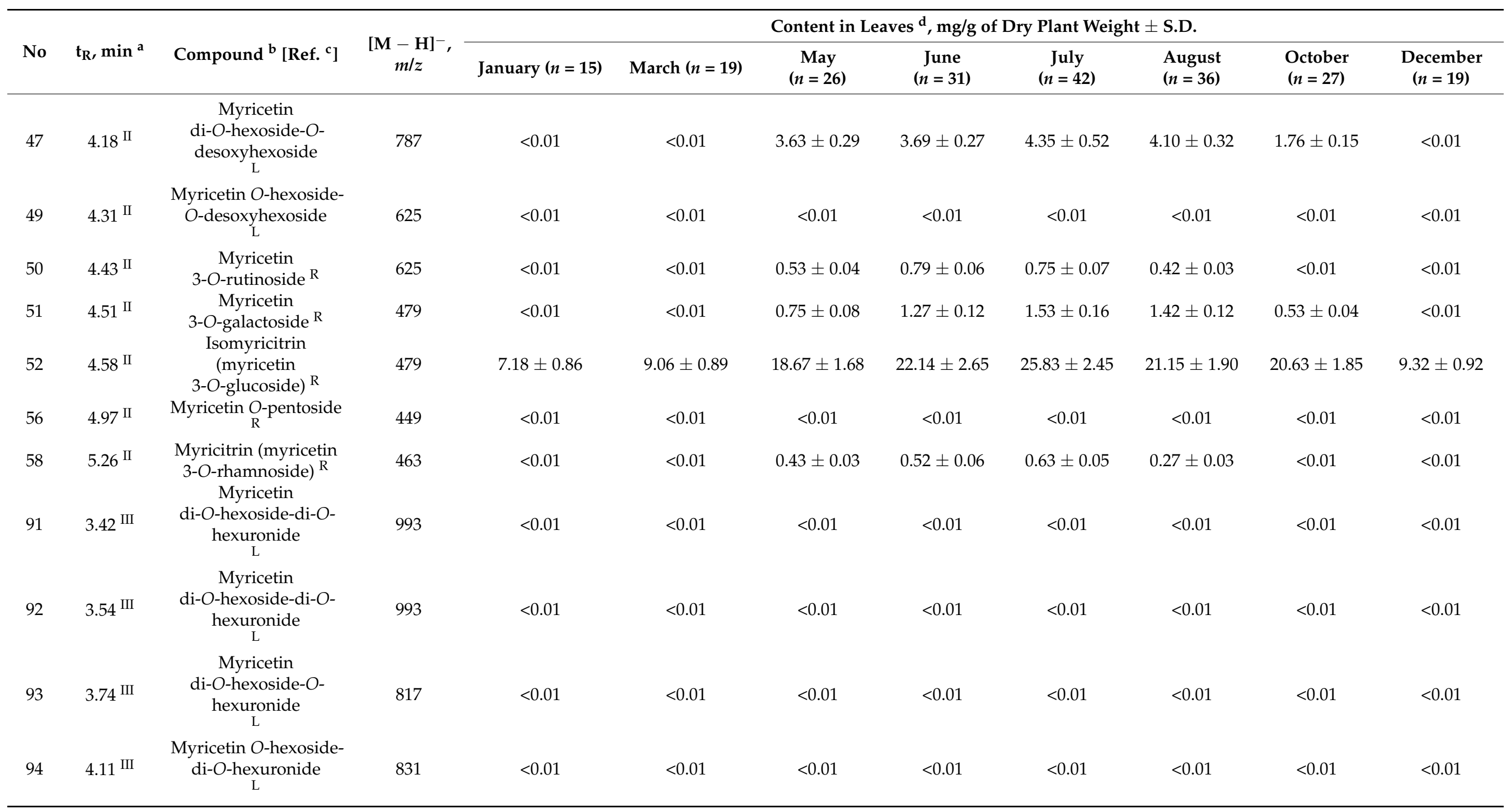


Table 3. Cont.

\begin{tabular}{|c|c|c|c|c|c|c|c|c|c|c|c|}
\hline \multirow[b]{2}{*}{ No } & \multirow[b]{2}{*}{$t_{R}, \min ^{a}$} & \multirow[b]{2}{*}{ Compound $^{b}$ [Ref. $\left.^{c}\right]$} & \multirow[b]{2}{*}{$\begin{array}{c}{[\mathbf{M}-\mathbf{H}]^{-}} \\
\quad m / z\end{array}$} & \multicolumn{8}{|c|}{ Content in Leaves ${ }^{\mathrm{d}}, \mathrm{mg} / \mathrm{g}$ of Dry Plant Weight \pm S.D. } \\
\hline & & & & January $(n=15)$ & March $(n=19)$ & $\begin{array}{c}\text { May } \\
(n=26)\end{array}$ & $\begin{array}{c}\text { June } \\
(n=31)\end{array}$ & $\begin{array}{c}\text { July } \\
(n=42)\end{array}$ & $\begin{array}{l}\text { August } \\
(n=36)\end{array}$ & $\begin{array}{l}\text { October } \\
(n=27)\end{array}$ & $\begin{array}{c}\text { December } \\
(n=19)\end{array}$ \\
\hline 97 & $4.67 \mathrm{III}$ & $\begin{array}{c}\text { Myricetin O-hexoside- } \\
\text { O-hexuronide } \\
\text { L }\end{array}$ & 655 & $2.63 \pm 0.31$ & $1.37 \pm 0.14$ & $6.40 \pm 0.70$ & $7.55 \pm 0.63$ & $9.32 \pm 0.74$ & $9.07 \pm 0.54$ & $5.18 \pm 0.41$ & $4.57 \pm 0.37$ \\
\hline 99 & $4.89 \mathrm{III}$ & $\begin{array}{c}\text { Myricetin } \\
\text { O-hexuronide }\end{array}$ & 493 & $4.76 \pm 0.30$ & $3.22 \pm 0.35$ & $8.26 \pm 0.75$ & $10.29 \pm 0.92$ & $11.57 \pm 1.23$ & $10.83 \pm 1.05$ & $8.62 \pm 0.73$ & $5.62 \pm 0.54$ \\
\hline 106 & $6.01 \mathrm{III}$ & $\begin{array}{c}\text { Myricetin O- } \\
\text { hexuronide-O-acetate } \\
\mathrm{L}\end{array}$ & 535 & $<0.01$ & $<0.01$ & $<0.01$ & $<0.01$ & $<0.01$ & $<0.01$ & $<0.01$ & $<0.01$ \\
\hline 107 & $6.78 \mathrm{III}$ & $\begin{array}{c}\text { Myricetin } O \text {-hexoside- } \\
\text { di-O-acetate } \\
\text { L }\end{array}$ & 563 & $<0.01$ & $<0.01$ & $<0.01$ & $<0.01$ & $<0.01$ & $<0.01$ & $<0.01$ & $<0.01$ \\
\hline 108 & $6.92^{\mathrm{III}}$ & $\begin{array}{c}\text { Myricetin } \\
\text { O-hexouronide-di-O- } \\
\text { acetate } \\
\text { L }\end{array}$ & 577 & $<0.01$ & $<0.01$ & $<0.01$ & $<0.01$ & $<0.01$ & $<0.01$ & $<0.01$ & $<0.01$ \\
\hline 111 & $7.92 \mathrm{III}$ & $\begin{array}{c}\text { Myricetin } O \text {-hexoside- } \\
\text { di-O-acetate } \\
\text { L }\end{array}$ & 563 & $<0.01$ & $<0.01$ & $<0.01$ & $<0.01$ & $<0.01$ & $<0.01$ & $<0.01$ & $<0.01$ \\
\hline 118 & $9.18 \mathrm{III}$ & $\begin{array}{c}\text { Myricetin } O \text {-hexoside- } \\
\text { tri-O-acetate } \\
\text { L }\end{array}$ & 605 & $<0.01$ & $<0.01$ & $<0.01$ & $<0.01$ & $<0.01$ & $<0.01$ & $<0.01$ & $<0.01$ \\
\hline 119 & $9.24 \mathrm{III}$ & $\begin{array}{c}\text { Myricetin } O \text {-hexoside- } \\
\text { tri-O-acetate } \\
\text { L }\end{array}$ & 605 & $<0.01$ & $<0.01$ & $<0.01$ & $<0.01$ & $<0.01$ & $<0.01$ & $0.53 \pm 0.03$ & $0.40 \pm 0.03$ \\
\hline 165 & 22.97 IV & $\begin{array}{c}\text { Myricetin tri-O- } \\
\text { hexoside-di-O-gallate } \\
\text { L }\end{array}$ & 1107 & $<0.01$ & $<0.01$ & $<0.01$ & $<0.01$ & $<0.01$ & $<0.01$ & $<0.01$ & $<0.01$ \\
\hline 166 & $23.67^{\mathrm{IV}}$ & $\begin{array}{c}\text { Myricetin di-O- } \\
\text { hexoside-di-O-gallate } \\
\text { L }\end{array}$ & 945 & $<0.01$ & $<0.01$ & $<0.01$ & $<0.01$ & $<0.01$ & $<0.01$ & $<0.01$ & $<0.01$ \\
\hline 167 & $24.43^{\mathrm{IV}}$ & $\begin{array}{c}\text { Myricetin di-O- } \\
\text { hexoside-O-gallate } \\
\text { L }\end{array}$ & 793 & $<0.01$ & $<0.01$ & $<0.01$ & $0.14 \pm 0.02$ & $0.37 \pm 0.03$ & $0.25 \pm 0.02$ & $<0.01$ & $<0.01$ \\
\hline
\end{tabular}


Table 3. Cont.

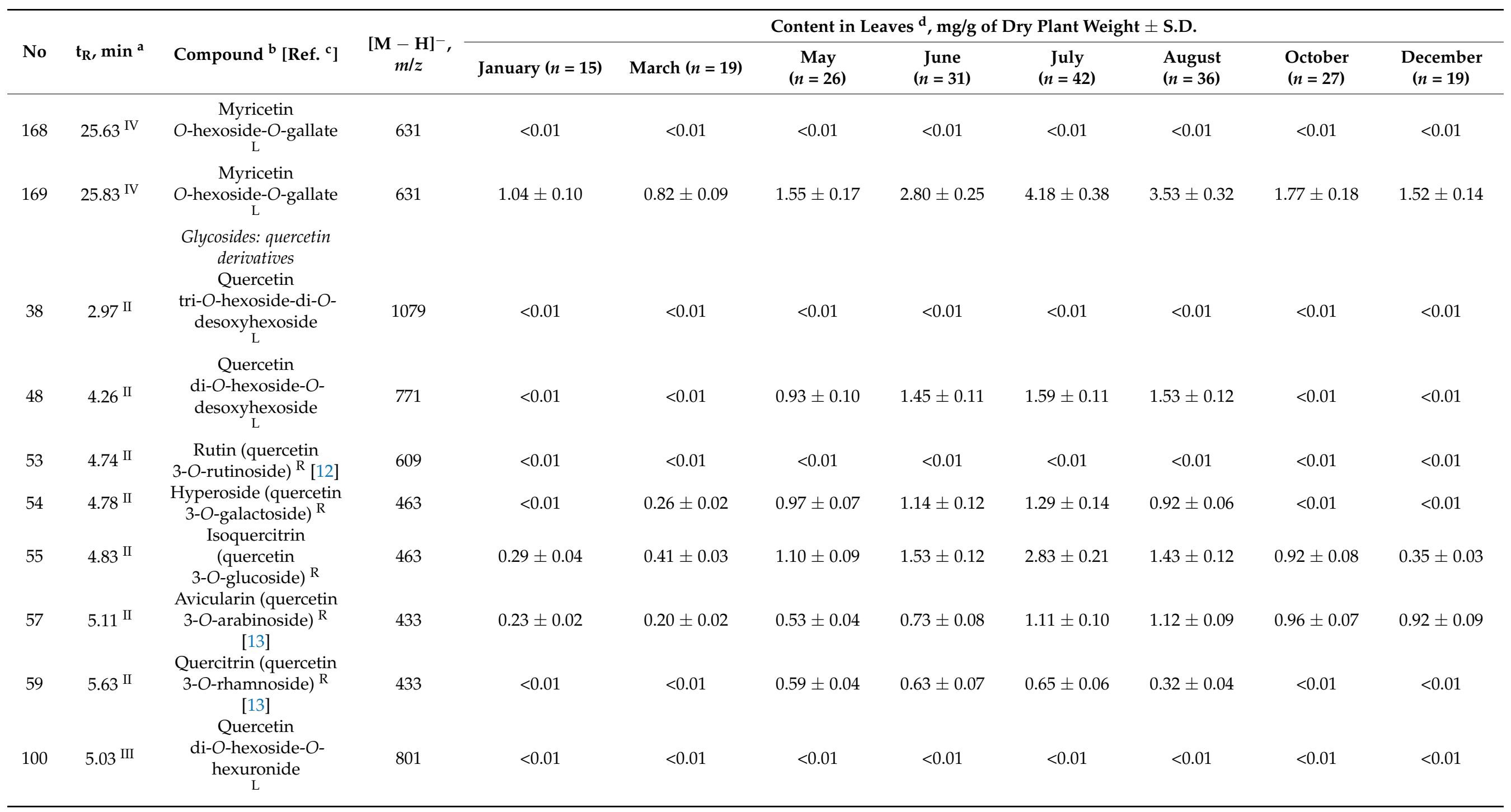


Table 3. Cont.

\begin{tabular}{|c|c|c|c|c|c|c|c|c|c|c|c|}
\hline \multirow[b]{2}{*}{ No } & \multirow[b]{2}{*}{$t_{R}, \min { }^{a}$} & \multirow[b]{2}{*}{ Compound $^{b}$ [Ref. $\left.^{c}\right]$} & \multirow[b]{2}{*}{$\begin{array}{l}{[\mathbf{M}-\mathbf{H}]^{-}} \\
\quad m / z\end{array}$} & \multicolumn{8}{|c|}{ Content in Leaves $^{\mathrm{d}}, \mathrm{mg} / \mathrm{g}$ of Dry Plant Weight \pm S.D. } \\
\hline & & & & January $(n=15)$ & $\operatorname{March}(n=19)$ & $\begin{array}{c}\text { May } \\
(n=26)\end{array}$ & $\begin{array}{c}\text { June } \\
(n=31)\end{array}$ & $\underset{(n=42)}{\text { July }}$ & $\begin{array}{l}\text { August } \\
(n=36)\end{array}$ & $\begin{array}{l}\text { October } \\
(n=27)\end{array}$ & $\begin{array}{c}\text { December } \\
(n=19)\end{array}$ \\
\hline 101 & $5.14 \mathrm{III}$ & $\begin{array}{l}\text { Quercetin O-hexoside- } \\
\text { di-O-hexuronide } \\
\text { L }\end{array}$ & 815 & $<0.01$ & $<0.01$ & $0.50 \pm 0.04$ & $0.53 \pm 0.04$ & $0.92 \pm 0.11$ & $0.86 \pm 0.12$ & $0.27 \pm 0.03$ & $<0.01$ \\
\hline 103 & $5.38 \mathrm{III}$ & $\begin{array}{l}\text { Quercetin O-hexoside- } \\
\text { O-hexuronide } \\
\text { L }\end{array}$ & 639 & $<0.01$ & $<0.01$ & $<0.01$ & $<0.01$ & $<0.01$ & $<0.01$ & $<0.01$ & $<0.01$ \\
\hline 105 & $5.69 \mathrm{III}$ & $\begin{array}{c}\text { Miquelianin } \\
\text { (quercetin }^{\text {quercos }} \\
\text { 3-Olucuronide) }\end{array}$ & 477 & $<0.01$ & $<0.01$ & $<0.01$ & $<0.01$ & $<0.01$ & $<0.01$ & $<0.01$ & $<0.01$ \\
\hline 110 & $7.36^{\mathrm{III}}$ & $\begin{array}{l}\text { Quercetin } O- \\
\text { hexuronide- } O \text {-acetate } \\
\text { L }\end{array}$ & 519 & $<0.01$ & $<0.01$ & $<0.01$ & $<0.01$ & $<0.01$ & $<0.01$ & $0.42 \pm 0.03$ & $0.37 \pm 0.03$ \\
\hline 114 & $8.47 \mathrm{III}$ & $\begin{array}{c}\text { Quercetin } \\
\text { O-hexuronide-di-O- } \\
\text { acetate } \\
\text { L }\end{array}$ & 561 & $<0.01$ & $<0.01$ & $<0.01$ & $<0.01$ & $<0.01$ & $<0.01$ & $<0.01$ & $<0.01$ \\
\hline 115 & $8.69 \mathrm{III}$ & $\begin{array}{c}\text { Quercetin } \\
\text { O-hexuronide-di-O- } \\
\text { acetate } \\
\text { L }\end{array}$ & 561 & $<0.01$ & $<0.01$ & $<0.01$ & $<0.01$ & $<0.01$ & $<0.01$ & $<0.01$ & $<0.01$ \\
\hline 121 & $9.46^{\mathrm{III}}$ & $\begin{array}{c}\text { Quercetin } O \text {-hexoside- } \\
\text { tri-O-acetate } \\
\text { L }\end{array}$ & 589 & $<0.01$ & $<0.01$ & $<0.01$ & $<0.01$ & $<0.01$ & $<0.01$ & $<0.01$ & $<0.01$ \\
\hline 122 & $9.53 \mathrm{III}$ & $\begin{array}{c}\text { Quercetin } O \text {-hexoside- } \\
\text { tri-O-acetate } \\
\text { L }\end{array}$ & 589 & $<0.01$ & $<0.01$ & $<0.01$ & $<0.01$ & $<0.01$ & $<0.01$ & $<0.01$ & $<0.01$ \\
\hline 170 & $26.74{ }^{\mathrm{IV}}$ & $\begin{array}{l}\text { Quercetin 3-O-(6"-O- } \\
\text { galloyl)-glucoside }\end{array}$ & 615 & $0.52 \pm 0.04$ & $0.50 \pm 0.05$ & $0.83 \pm 0.06$ & $1.07 \pm 0.11$ & $1.54 \pm 0.12$ & $1.62 \pm 0.14$ & $0.93 \pm 0.10$ & $0.73 \pm 0.06$ \\
\hline 171 & $28.02^{\mathrm{IV}}$ & $\begin{array}{l}\text { Quercetin O-hexoside- } \\
\text { di-O-gallate }\end{array}$ & 767 & $<0.01$ & $<0.01$ & $<0.01$ & $0.53 \pm 0.04$ & $0.96 \pm 0.10$ & $0.83 \pm 0.09$ & $0.21 \pm 0.02$ & $<0.01$ \\
\hline
\end{tabular}


Table 3. Cont.




Table 3. Cont.

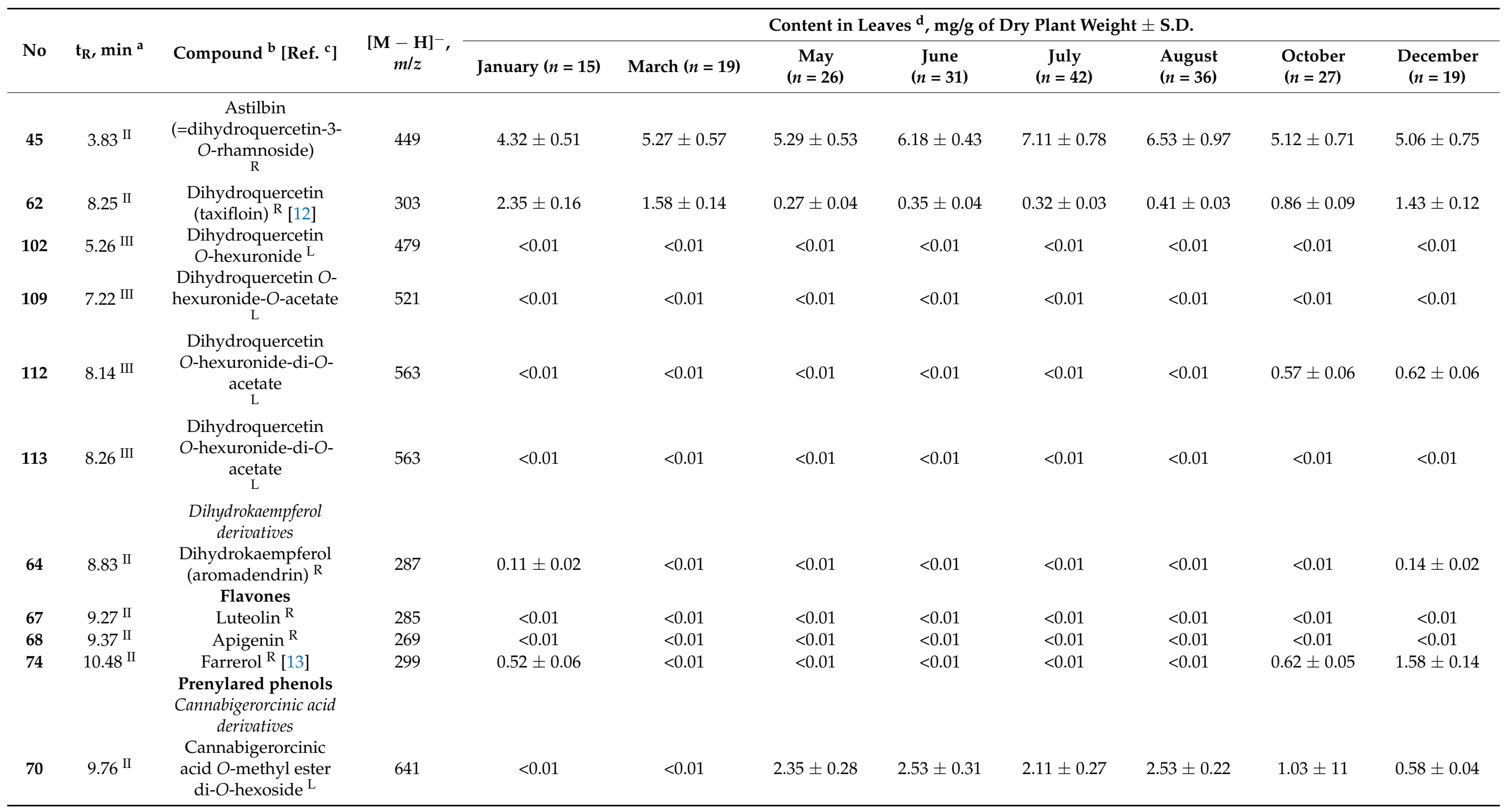


Table 3. Cont.

\begin{tabular}{|c|c|c|c|c|c|c|c|c|c|c|c|}
\hline \multirow[b]{2}{*}{ No } & \multirow[b]{2}{*}{$t_{R}, \min ^{a}$} & \multirow[b]{2}{*}{ Compound $^{b}\left[\right.$ Ref. $\left.^{c}\right]$} & \multirow[b]{2}{*}{$\begin{array}{c}{[\mathbf{M}-\mathbf{H}]^{-}} \\
\quad m / z\end{array}$} & \multicolumn{8}{|c|}{ Content in Leaves $^{\mathrm{d}}, \mathrm{mg} / \mathrm{g}$ of Dry Plant Weight \pm S.D. } \\
\hline & & & & January $(n=15)$ & March $(n=19)$ & $\begin{array}{c}\text { May } \\
(n=26)\end{array}$ & $\begin{array}{c}\text { June } \\
(n=31)\end{array}$ & $\underset{(n=42)}{\text { July }}$ & $\begin{array}{l}\text { August } \\
(n=36)\end{array}$ & $\begin{array}{l}\text { October } \\
(n=27)\end{array}$ & $\begin{array}{c}\text { December } \\
(n=19)\end{array}$ \\
\hline 71 & $9.93 \mathrm{II}$ & $\begin{array}{l}\text { Cannabigerorcinic } \\
\text { acid } O \text {-methyl ester } \\
\text { di-O-hexoside }{ }^{\mathrm{L}}\end{array}$ & 641 & $<0.01$ & $<0.01$ & $0.62 \pm 0.05$ & $0.69 \pm 0.06$ & $0.50 \pm 0.04$ & $0.31 \pm 0.02$ & $<0.01$ & $<0.01$ \\
\hline 72 & $10.04^{\mathrm{II}}$ & $\begin{array}{c}\text { Cannabigerorcinic } \\
\text { acid O-methyl ester } \\
O \text {-hexoside-O- } \\
\text { desoxyhexoside } \\
\text { L }\end{array}$ & 625 & $<0.01$ & $<0.01$ & $0.95 \pm 0.10$ & $1.14 \pm 0.12$ & $1.16 \pm 0.14$ & $0.73 \pm 0.08$ & $<0.01$ & $<0.01$ \\
\hline 75 & 10.63 II & $\begin{array}{l}\text { Cannabigerorcinic } \\
\text { acid methyl ester } \\
\text { O-hexoside }\end{array}$ & 479 & $1.43 \pm 0.12$ & $1.20 \pm 0.10$ & $1.93 \pm 0.22$ & $2.35 \pm 0.25$ & $2.30 \pm 0.23$ & $2.04 \pm 0.20$ & $2.56 \pm 0.24$ & $2.33 \pm 0.20$ \\
\hline 76 & $10.97^{\text {II }}$ & $\begin{array}{c}\text { Cannabigerorcinic } \\
\text { acid di-O-methyl ester } \\
\text { O-hexoside } \mathrm{L}\end{array}$ & 493 & $<0.01$ & $<0.01$ & $<0.01$ & $<0.01$ & $<0.01$ & $<0.01$ & $<0.01$ & $<0.01$ \\
\hline 77 & $11.05^{\mathrm{II}}$ & $\begin{array}{c}\text { Cannabigerorcinic } \\
\text { acid di-O-methyl ester } \\
\text { O-hexoside }{ }^{\mathrm{L}}\end{array}$ & 493 & $<0.01$ & $<0.01$ & $<0.01$ & $<0.01$ & $<0.01$ & $<0.01$ & $<0.01$ & $<0.01$ \\
\hline 78 & 11.22 II & $\begin{array}{c}\text { Cannabigerorcinic } \\
\text { acid di-O-methyl ester } \\
\text { O-hexoside }\end{array}$ & 493 & $<0.01$ & $<0.01$ & $<0.01$ & $<0.01$ & $<0.01$ & $<0.01$ & $<0.01$ & $<0.01$ \\
\hline 79 & $11.47^{\mathrm{II}}$ & $\begin{array}{l}\text { Cannabigerorcinic } \\
\text { acid } O \text {-methyl ester } \mathrm{L}\end{array}$ & 317 & $35.16 \pm 3.57$ & $32.03 \pm 3.28$ & $18.35 \pm 2.14$ & $20.39 \pm 2.24$ & $19.03 \pm 1.92$ & $25.76 \pm 2.06$ & $36.18 \pm 3.25$ & $39.92 \pm 4.02$ \\
\hline 80 & $11.52^{\mathrm{II}}$ & $\begin{array}{l}\text { Cannabigerorcinic } \\
\text { acid } O \text {-methyl ester }{ }^{\mathrm{L}}\end{array}$ & 317 & $0.14 \pm 0.02$ & $<0.01$ & $<0.01$ & $<0.01$ & $<0.01$ & $<0.01$ & $<0.01$ & $0.24 \pm 0.02$ \\
\hline 81 & $11.74^{\mathrm{II}}$ & $\begin{array}{l}\text { Cannabigerorcinic } \\
\text { acid di-O-methyl ester } \\
\text { L }\end{array}$ & 331 & $0.10 \pm 0.02$ & $<0.01$ & $<0.01$ & $<0.01$ & $<0.01$ & $<0.01$ & $<0.01$ & $0.10 \pm 0.01$ \\
\hline 82 & $12.04^{\mathrm{II}}$ & $\begin{array}{c}\text { Cannabigerorcinic } \\
\text { acid di-O-methyl ester } \\
\text { L }\end{array}$ & 331 & $0.20 \pm 0.02$ & $<0.01$ & $<0.01$ & $<0.01$ & $<0.01$ & $<0.01$ & $<0.01$ & $0.18 \pm 0.02$ \\
\hline 83 & $13.15^{\text {II }}$ & $\begin{array}{c}\text { Cannabigerorcinic } \\
\text { acid tri-O-methyl ester } \\
\mathrm{L}\end{array}$ & 345 & $0.18 \pm 0.03$ & $<0.01$ & $<0.01$ & $<0.01$ & $<0.01$ & $<0.01$ & $<0.01$ & $0.27 \pm 0.03$ \\
\hline 116 & $8.92 \mathrm{III}$ & $\begin{array}{l}\text { Cannabigerorcinic } \\
\text { acid di-O-hexoside }^{\mathrm{L}}\end{array}$ & 627 & $<0.01$ & $<0.01$ & $<0.01$ & $<0.01$ & $<0.01$ & $<0.01$ & $<0.01$ & $<0.01$ \\
\hline
\end{tabular}


Table 3. Cont.

\begin{tabular}{|c|c|c|c|c|c|c|c|c|c|c|c|}
\hline \multirow[b]{2}{*}{ No } & \multirow[b]{2}{*}{$t_{R}, \min ^{a}$} & \multirow[b]{2}{*}{ Compound $^{b}\left[\right.$ Ref. $\left.^{c}\right]$} & \multirow[b]{2}{*}{$\begin{array}{l}{[\mathbf{M}-\mathbf{H}]^{-}} \\
\quad m / z\end{array}$} & \multicolumn{8}{|c|}{ Content in Leaves $^{d}, \mathrm{mg} / \mathrm{g}$ of Dry Plant Weight \pm S.D. } \\
\hline & & & & January $(n=15)$ & March $(n=19)$ & $\begin{array}{c}\text { May } \\
(n=26)\end{array}$ & $\underset{(n=31)}{\text { June }}$ & $\underset{(n=42)}{\text { July }}$ & $\begin{array}{l}\text { August } \\
(n=36)\end{array}$ & $\begin{array}{l}\text { October } \\
(n=27)\end{array}$ & $\begin{array}{c}\text { December } \\
(n=19)\end{array}$ \\
\hline 117 & $9.01 \mathrm{III}$ & $\begin{array}{l}\text { Cannabigerorcinic } \\
\text { acid } O \text {-hexoside }^{\mathrm{L}}\end{array}$ & 465 & $0.40 \pm 0.04$ & $<0.01$ & $<0.01$ & $<0.01$ & $<0.01$ & $<0.01$ & $0.92 \pm 0.11$ & $0.86 \pm 0.09$ \\
\hline 120 & $9.33 \mathrm{III}$ & $\begin{array}{l}\text { Cannabigerorcinic } \\
\text { acid }^{\mathrm{R}}[13]\end{array}$ & 303 & $0.63 \pm 0.07$ & $0.62 \pm 0.08$ & $0.37 \pm 0.04$ & $0.21 \pm 0.02$ & $0.08 \pm 0.00$ & $0.21 \pm 0.03$ & $0.95 \pm 0.11$ & $0.99 \pm 0.11$ \\
\hline 123 & $9.72 \mathrm{III}$ & $\begin{array}{l}\text { Cannabigerorcinic } \\
\text { acid } O \text {-acetate }\end{array}$ & 345 & $0.34 \pm 0.03$ & $0.19 \pm 0.02$ & $<0.01$ & $<0.01$ & $<0.01$ & $<0.01$ & $0.42 \pm 0.03$ & $0.40 \pm 0.03$ \\
\hline 124 & $9.81 \mathrm{III}$ & $\begin{array}{l}\text { Cannabigerorcinic } \\
\text { acid di-O-acetate }\end{array}$ & 387 & $1.05 \pm 0.09$ & $0.86 \pm 0.07$ & $<0.01$ & $<0.01$ & $<0.01$ & $0.38 \pm 0.04$ & $1.53 \pm 0.14$ & $1.27 \pm 0.11$ \\
\hline 129 & $10.40 \mathrm{III}$ & $\begin{array}{l}\text { Cannabigerorcinic } \\
\text { acid tri-O-acetate }\end{array}$ & 429 & $<0.01$ & $<0.01$ & $<0.01$ & $<0.01$ & $<0.01$ & $<0.01$ & $<0.01$ & $<0.01$ \\
\hline 125 & $9.93 \mathrm{III}$ & $\begin{array}{l}\text { Grifolic acid derivatives } \\
\text { Hydroxy-grifolic acid } \\
\text { di-O-hexoside }\end{array}$ & 711 & $<0.01$ & $<0.01$ & $<0.01$ & $<0.01$ & $<0.01$ & $0.53 \pm 0.06$ & $0.62 \pm 0.05$ & $0.35 \pm 0.04$ \\
\hline 126 & $9.98 \mathrm{III}$ & $\begin{array}{c}\text { Hydroxy-grifolic acid } \\
\text { O-hexoside }\end{array}$ & 549 & $0.42 \pm 0.04$ & $0.27 \pm 0.04$ & $1.22 \pm 0.10$ & $1.53 \pm 0.16$ & $2.14 \pm 0.19$ & $2.53 \pm 0.22$ & $1.67 \pm 0.14$ & $0.93 \pm 0.10$ \\
\hline 127 & $10.09 \mathrm{III}$ & $\begin{array}{c}\text { Hydroxy-grifolic acid } \\
\text { O-hexoside }\end{array}$ & 549 & $0.97 \pm 0.11$ & $0.53 \pm 0.04$ & $1.09 \pm 0.12$ & $2.75 \pm 0.24$ & $3.10 \pm 0.31$ & $3.16 \pm 0.28$ & $2.39 \pm 0.22$ & $1.86 \pm 0.16$ \\
\hline 128 & $10.27^{\mathrm{III}}$ & $\begin{array}{c}\text { Hydroxy-grifolic acid } \\
\text { O-pentoside }\end{array}$ & 519 & $0.11 \pm 0.02$ & $<0.01$ & $<0.01$ & $0.20 \pm 0.01$ & $0.63 \pm 0.04$ & $1.45 \pm 0.10$ & $0.92 \pm 0.11$ & $0.53 \pm 0.04$ \\
\hline 130 & $10.67 \mathrm{III}$ & Hydroxy-grifolic acid & 387 & $3.15 \pm 0.40$ & $3.01 \pm 0.39$ & $1.86 \pm 0.20$ & $1.04 \pm 0.11$ & $1.59 \pm 0.14$ & $3.67 \pm 0.34$ & $4.18 \pm 0.39$ & $4.50 \pm 0.48$ \\
\hline 131 & $10.75 \mathrm{III}$ & $\begin{array}{c}\text { Grifolic acid } \\
\text { di-O-hexoside }\end{array}$ & 695 & $<0.01$ & $<0.01$ & $<0.01$ & $<0.01$ & $<0.01$ & $<0.01$ & $<0.01$ & $<0.01$ \\
\hline 132 & $10.86^{\mathrm{III}}$ & $\begin{array}{l}\text { Grifolic acid } \\
\text { O-hexoside }\end{array}$ & 533 & $<0.01$ & $<0.01$ & $<0.01$ & $<0.01$ & $0.27 \pm 0.03$ & $0.50 \pm 0.03$ & $0.42 \pm 0.04$ & $0.22 \pm 0.03$ \\
\hline 137 & $11.64^{\mathrm{III}}$ & Grifolic acid $^{\mathrm{R}}$ & 371 & $7.09 \pm 0.67$ & $6.59 \pm 0.65$ & $4.18 \pm 0.42$ & $3.62 \pm 0.40$ & $5.73 \pm 0.52$ & $5.62 \pm 0.57$ & $7.33 \pm 0.69$ & $7.56 \pm 0.73$ \\
\hline 138 & $12.72 \mathrm{III}$ & $\begin{array}{c}\text { Grifolic acid } \mathrm{O} \text {-methyl } \\
\text { ester } \mathrm{L}\end{array}$ & 385 & $<0.01$ & $<0.01$ & $<0.01$ & $<0.01$ & $<0.01$ & $<0.01$ & $<0.01$ & $<0.01$ \\
\hline 139 & $12.81^{\mathrm{III}}$ & $\begin{array}{c}\text { Grifolic acid } \\
\text { di-O-methyl ester L }\end{array}$ & 399 & $<0.01$ & $<0.01$ & $<0.01$ & $<0.01$ & $<0.01$ & $<0.01$ & $<0.01$ & $<0.01$ \\
\hline 140 & $12.92 \mathrm{III}$ & $\begin{array}{l}\text { Grifolic acid O-methyl } \\
\text { ester-O-acetate } \mathrm{L} \\
\text { Daurichromenic acid } \\
\text { derivatives }\end{array}$ & 427 & $<0.01$ & $<0.01$ & $<0.01$ & $<0.01$ & $<0.01$ & $<0.01$ & $<0.01$ & $<0.01$ \\
\hline
\end{tabular}


Table 3. Cont.

\begin{tabular}{|c|c|c|c|c|c|c|c|c|c|c|c|}
\hline \multirow[b]{2}{*}{ No } & \multirow[b]{2}{*}{$t_{R}, \min ^{a}$} & \multirow[b]{2}{*}{ Compound $^{b}\left[\right.$ Ref. $\left.^{c}\right]$} & \multirow[b]{2}{*}{$\begin{array}{l}{[\mathbf{M}-\mathbf{H}]^{-}} \\
\quad m / z\end{array}$} & \multicolumn{8}{|c|}{ Content in Leaves $^{\mathrm{d}}, \mathrm{mg} / \mathrm{g}$ of Dry Plant Weight \pm S.D. } \\
\hline & & & & January $(n=15)$ & $\operatorname{March}(n=19)$ & $\begin{array}{c}\text { May } \\
(n=26)\end{array}$ & $\begin{array}{c}\text { June } \\
(n=31)\end{array}$ & $\underset{(n=42)}{\text { July }}$ & $\begin{array}{l}\text { August } \\
(n=36)\end{array}$ & $\begin{array}{l}\text { October } \\
(n=27)\end{array}$ & $\begin{array}{c}\text { December } \\
(n=19)\end{array}$ \\
\hline 133 & $10.86^{\mathrm{III}}$ & $\begin{array}{c}\text { Daurichromenic acid } \\
\text { di-O-hexoside }\end{array}$ & 693 & $<0.01$ & $<0.01$ & $<0.01$ & $<0.01$ & $<0.01$ & $<0.01$ & $<0.01$ & $<0.01$ \\
\hline 134 & $11.06^{\mathrm{III}}$ & $\begin{array}{c}\text { Daurichromenic acid } \\
\text { O-hexoside }\end{array}$ & 531 & $<0.01$ & $<0.01$ & $<0.01$ & $<0.01$ & $<0.01$ & $<0.01$ & $<0.01$ & $<0.01$ \\
\hline 135 & $11.26^{\mathrm{III}}$ & 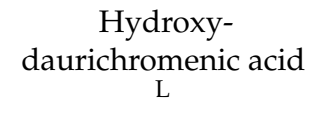 & 385 & $3.57 \pm 0.33$ & $3.02 \pm 0.31$ & $0.25 \pm 0.03$ & $0.77 \pm 0.08$ & $0.93 \pm 0.11$ & $2.09 \pm 0.21$ & $2.59 \pm 0.31$ & $3.82 \pm 0.35$ \\
\hline 136 & $11.43^{\mathrm{III}}$ & $\begin{array}{c}\text { Hydroxy- } \\
\text { daurichromenic acid } \\
\text { O-methyl ester }{ }^{\mathrm{L}}\end{array}$ & 399 & $1.63 \pm 0.17$ & $1.42 \pm 0.12$ & $<0.01$ & $<0.01$ & $0.50 \pm 0.04$ & $0.84 \pm 0.07$ & $1.42 \pm 0.10$ & $1.53 \pm 0.14$ \\
\hline 141 & $13.14^{\mathrm{III}}$ & $\begin{array}{c}\text { Daurichromenic acid } \\
\mathrm{R}_{[}[13]\end{array}$ & 369 & $2.30 \pm 0.21$ & $1.93 \pm 0.20$ & $1.04 \pm 0.09$ & $1.27 \pm 0.14$ & $1.53 \pm 0.14$ & $2.07 \pm 0.17$ & $2.56 \pm 0.22$ & $2.69 \pm 0.25$ \\
\hline 142 & $13.42^{\mathrm{III}}$ & $\begin{array}{c}\text { Daurichromenic acid } \\
\text { O-acetate }\end{array}$ & 411 & $<0.01$ & $<0.01$ & $<0.01$ & $<0.01$ & $<0.01$ & $<0.01$ & $<0.01$ & $<0.01$ \\
\hline 143 & $13.58^{\mathrm{III}}$ & $\begin{array}{l}\text { Daurichromenic acid } \\
\text { O-methyl ester } \mathrm{L}\end{array}$ & 383 & $<0.01$ & $<0.01$ & $<0.01$ & $<0.01$ & $<0.01$ & $<0.01$ & $<0.01$ & $<0.01$ \\
\hline 145 & $14.23 \mathrm{III}$ & $\begin{array}{c}\text { Daurichromenic acid } \\
\text { di-O-methyl ester }{ }^{\mathrm{L}} \\
\text { Benzoic acid } \\
\text { derivatives }\end{array}$ & 397 & $<0.01$ & $<0.01$ & $<0.01$ & $<0.01$ & $<0.01$ & $<0.01$ & $<0.01$ & $<0.01$ \\
\hline 84 & $0.68^{\mathrm{III}}$ & $\begin{array}{l}\text { Protocatechuic acid } \\
\text { di-O-hexoside }\end{array}$ & 477 & $<0.01$ & $<0.01$ & $<0.01$ & $0.14 \pm 0.01$ & $0.53 \pm 0.04$ & $0.63 \pm 0.05$ & $0.21 \pm 0.02$ & $<0.01$ \\
\hline 85 & $0.89 \mathrm{III}$ & $\begin{array}{l}\text { Protocatechuic acid } \\
\text { O-hexoside } \mathrm{L}^{\mathrm{L}}\end{array}$ & 315 & $<0.01$ & $<0.01$ & $<0.01$ & $<0.01$ & $0.23 \pm 0.02$ & $<0.01$ & $<0.01$ & $<0.01$ \\
\hline 87 & $2.71 \mathrm{III}$ & $\begin{array}{l}\text { Vanillic/isovanillic } \\
\text { acid } O \text {-hexoside }\end{array}$ & 329 & $<0.01$ & $<0.01$ & $<0.01$ & $<0.01$ & $<0.01$ & $<0.01$ & $<0.01$ & $<0.01$ \\
\hline 88 & $2.76^{\mathrm{III}}$ & $\begin{array}{l}\text { Vanillic/isovanillic } \\
\text { acid } O \text {-hexoside }{ }^{\mathrm{L}}\end{array}$ & 329 & $<0.01$ & $<0.01$ & $<0.01$ & $<0.01$ & $<0.01$ & $<0.01$ & $<0.01$ & $<0.01$ \\
\hline 89 & $2.81^{\mathrm{III}}$ & $\begin{array}{c}\text { Vanillic acid } \\
\text { 4-O-glucoside }\end{array}$ & 329 & $<0.01$ & $<0.01$ & $0.53 \pm 0.06$ & $1.90 \pm 0.16$ & $2.51 \pm 0.21$ & $2.07 \pm 0.20$ & $0.95 \pm 0.10$ & $0.27 \pm 0.03$ \\
\hline 90 & $2.95^{\mathrm{III}}$ & $\begin{array}{l}\text { Vanillic/isovanillic } \\
\text { acid } O \text {-hexoside } \mathrm{L}\end{array}$ & 329 & $<0.01$ & $<0.01$ & $<0.01$ & $<0.01$ & $<0.01$ & $<0.01$ & $<0.01$ & $<0.01$ \\
\hline
\end{tabular}


Table 3. Cont.

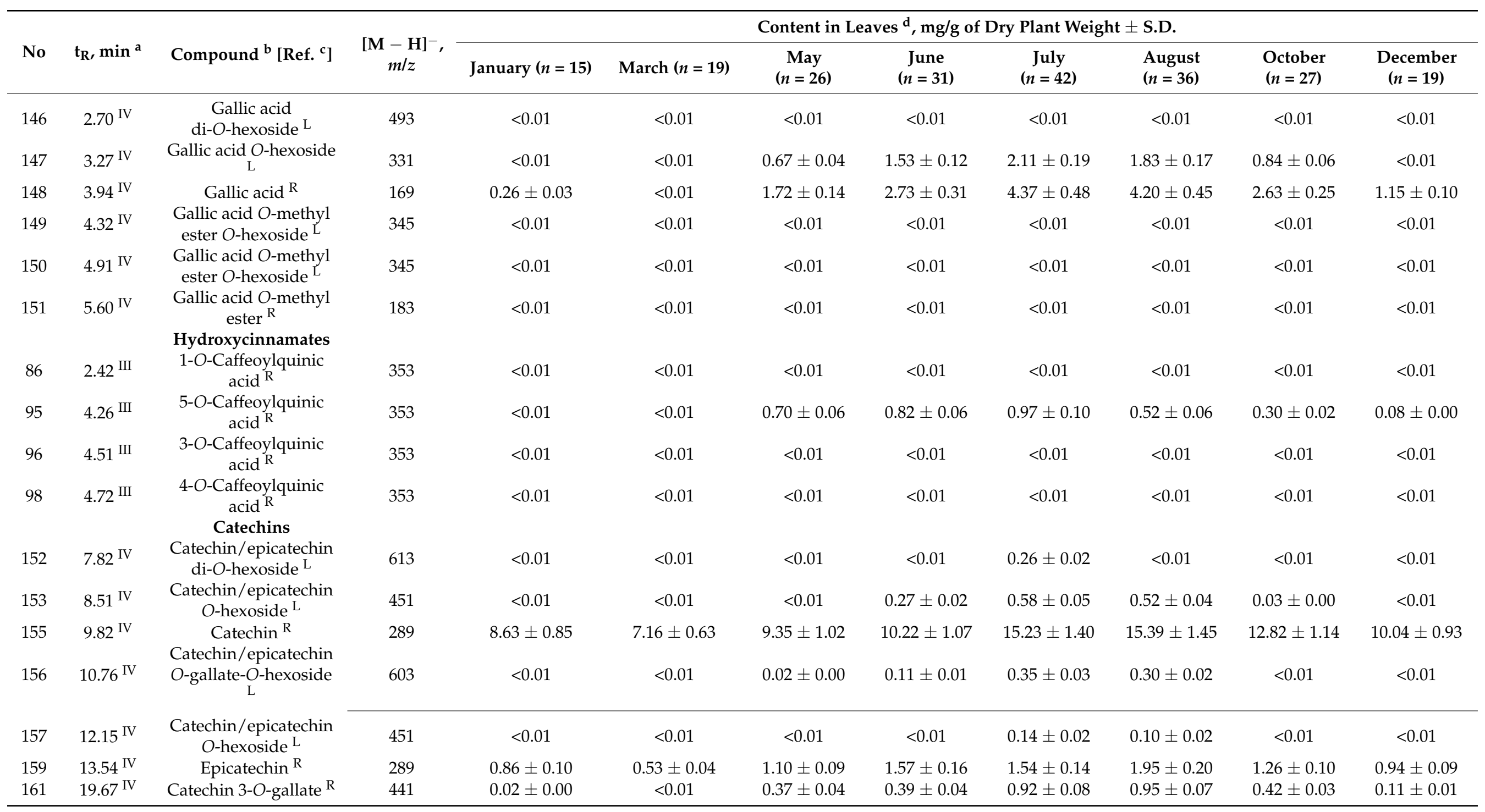


Table 3. Cont

\begin{tabular}{|c|c|c|c|c|c|c|c|c|c|c|c|}
\hline \multirow[b]{2}{*}{ No } & \multirow[b]{2}{*}{$t_{R}, \min ^{a}$} & \multirow[b]{2}{*}{ Compound $^{b}\left[\right.$ Ref. $\left.^{c}\right]$} & \multirow[b]{2}{*}{$\begin{array}{c}{[\mathbf{M}-\mathbf{H}]^{-}} \\
\quad m / z\end{array}$} & \multicolumn{8}{|c|}{ Content in Leaves ${ }^{\mathrm{d}}, \mathrm{mg} / \mathrm{g}$ of Dry Plant Weight \pm S.D. } \\
\hline & & & & January $(n=15)$ & March $(n=19)$ & $\begin{array}{c}\text { May } \\
(n=26)\end{array}$ & $\underset{(n=31)}{\text { June }}$ & $\underset{(n=42)}{\text { July }}$ & $\begin{array}{l}\text { August } \\
(n=36)\end{array}$ & $\begin{array}{l}\text { October } \\
(n=27)\end{array}$ & $\begin{array}{c}\text { December } \\
(n=19)\end{array}$ \\
\hline 164 & $22.26^{\mathrm{IV}}$ & $\begin{array}{l}\text { Epicatechin } \\
\text { 3-O-gallate R } \\
\text { Procyanidins }\end{array}$ & 441 & $<0.01$ & $<0.01$ & $<0.01$ & $<0.01$ & $<0.01$ & $<0.01$ & $<0.01$ & $<0.01$ \\
\hline 154 & $9.03 \mathrm{IV}$ & Procyanidin $B_{1} R$ & 577 & $0.95 \pm 0.08$ & $0.73 \pm 0.08$ & $1.14 \pm 0.10$ & $2.06 \pm 0.018$ & $2.89 \pm 0.25$ & $2.73 \pm 0.26$ & $1.39 \pm 0.14$ & $1.22 \pm 0.10$ \\
\hline 158 & $13.02 \mathrm{IV}$ & Procyanidin $B_{2} R$ & 577 & $<0.01$ & $<0.01$ & $<0.01$ & $<0.01$ & $<0.01$ & $<0.01$ & $<0.01$ & $<0.01$ \\
\hline 160 & 15.51 IV & Procyanidin $C_{1} R$ & 865 & $0.72 \pm 0.06$ & $0.63 \pm 0.06$ & $0.99 \pm 0.10$ & $1.27 \pm 0.14$ & $1.37 \pm 0.14$ & $1.27 \pm 0.11$ & $1.02 \pm 0.09$ & $0.83 \pm 0.09$ \\
\hline 162 & $20.52 \mathrm{IV}$ & $\begin{array}{l}\text { Catechin/epicatechin } \\
\text { dimer O-gallate } \mathrm{L}\end{array}$ & 729 & $<0.01$ & $<0.01$ & $<0.01$ & $<0.01$ & $<0.01$ & $<0.01$ & $<0.01$ & $<0.01$ \\
\hline 163 & $21.48^{\mathrm{IV}}$ & $\begin{array}{l}\text { Catechin/epicatechin } \\
\text { dimer di-O-gallate } \mathrm{L} \\
\text { Dihydrochalcones }\end{array}$ & 881 & $<0.01$ & $<0.01$ & $<0.01$ & $<0.01$ & $<0.01$ & $<0.01$ & $<0.01$ & $<0.01$ \\
\hline 63 & $8.68^{\mathrm{II}}$ & Phloretin ${ }^{R}$ & 273 & $0.18 \pm 0.02$ & $<0.01$ & $<0.01$ & $<0.01$ & $<0.01$ & $<0.01$ & $0.23 \pm 0.02$ & $0.29 \pm 0.03$ \\
\hline
\end{tabular}

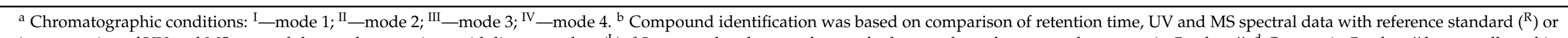

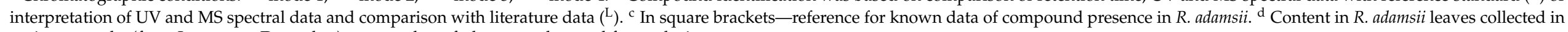
various months (from January to December). $n$-number of plant samples used for analysis. 


\subsubsection{Carbohydrates and Organic Acids}

The highly hydrophilic compounds of $R$. adamsii leaves comprised two carbohydratesO-hexosyl-hexose (1) and hexose (2), and five organic acids-malic (3), citric (4), tartaric (5), succinic (6), and fumaric acids (7). The nature of the carbohydrates was studied using high-performance anion-exchange chromatography [56], which showed the presence of saccharose, raffinose, glucose, fructose, and galactose (Figure S1).

\subsubsection{Simple Phenol Glycosides}

Fifteen phenolic glycosides were found in R. adamsii, mostly derivatives of phloroglucinol (8-14), hydroquinone $(15-17,19,20)$, and orcinol $(18,21,24)$. Phloroglucinols were in the form of di-O-hexosides $(\mathbf{8}, \mathbf{9}), \mathrm{O}$-glucoside (phlorin, 10$)$, di-O-hexoside- $\mathrm{O}$-acetates $(11,12)$, and di-O-hexoside-di-O-acetates $(13,14)$. Previous data regarding phloroglucinol presence in the Rhododendron genus includes 1-O-acetylphloroglucinol found in R. ferrugineum [44], therefore, phloroglucinol glycosides were detected here for the first time in the genus. The hydroquinone glycoside, arbutin (17), typical for some Ericaceae plants [45], was identified in $R$. adamsii using a reference standard; this compound had previously been detected in $R$. latoucheae [57] and R. arboreum [58]. Some rare hydroquinone glycosides were discovered, such as hydroquinone di-O-hexosides $(15,16)$ with the possible structure of known 6-O-glucosyl arbutin [45], an unknown hydroquinone di-O-hexoside$O$-methyl ester (19), and a hydroquinone $O$-hexoside- $O$-methyl ester (20) that is probably methylarbutin [46].

Sakakin (21), the orcinol $O$-glucoside that was identified after comparison with the reference standard, was found in rhododendrons for the first time, although orcinol itself has previously been detected in $R$. dauricum twigs [47]. Additionally, orcinol di-O-hexoside (18) and its $O$-acetate (24) were components of $R$. adamsii leaves. The unusual combination of simple phenolic glycosides in R. adamsii probably relates to its specific metabolomic features, which are extraordinary for the Rhododendron genus but understandable for the Ericaceae family.

\subsubsection{Triterpene Glycosides}

Preliminary analysis of SPE-1 eluate using acidic hydrolysis followed by HPLC separation allowed the detection of ursolic acid and unquantifiable traces of oleanolic acid (Figure S2). The use of the LC-MS technique showed thirteen ursolic acid derivatives as tri- $O$-hexosides $(22,23)$, di-O-hexosides $(\mathbf{2 5}, \mathbf{2 6})$, tri- $O$-hexoside- $O$-acetates $(\mathbf{2 7}, \mathbf{2 8})$, di-O-hexoside- $O$-acetate (29), $O$-hexoside (30), di-O-hexoside-di-O-acetates (31, 32), $O$ hexoside- $O$-acetates $(33,34)$, and $O$-hexoside-di-O-acetate (35) in the $R$. adamsii leaves. The results of earlier studies of $R$. adamsii $[59,60]$ demonstrated the presence of a low level of free ursolic acid $(<1 \mathrm{mg} / \mathrm{g})$, owing to the domination of the glycosylated form of triterpene acids in the plant.

\subsubsection{Flavonoids}

Sixty-eight compounds found in various SPE eluates of $R$. adamsii leaves extract were determined as flavonoids and separated into flavonols (52 compounds), dihydroflavonols (13 compounds), and flavones ( 3 compounds) in glycoside and aglycone states.

Flavonols belonged to one of three groups depending on their aglycone structuremyricetin, quercetin, or kaempferol. The myricetin group was the largest, with twentyseven members including neutral, acidic, and acylated derivatives. After comparison with reference standards, some neutral myricetins were identified as myricetin-3-O-rutinoside (50), myricetin-3-O-galactoside (51), myricetin-3-O-glucoside (isomyricitrin, 52), and myricetin-3-Orhamnoside (myricitrin, 58). The remaining compounds observed in mass spectra the loss of pentose fragments with $m / z 132$ (myricetin O-pentoside, 56) or fragments of hexose $(\mathrm{m} / \mathrm{z}$ 162) and desoxyhexose $(\mathrm{m} / \mathrm{z} 146)$ in ratios of 1:1 (myricetin $O$-hexoside- $O$-desoxyhexoside, 49), 2:1 (myricetin di-O-hexoside-O-desoxyhexoside, 47), 2:2 (myricetin di-O-hexoside- 
di-O-desoxyhexoside, 46), 3:2 (myricetin tri-O-hexoside-di-O-desoxyhexoside, 37), or 3:3 (myricetin tri-O-hexoside-tri-O-desoxyhexoside, 36).

The known analogs of 56 are myricetin-3-O-arabinoside and myricetin-3-O-xyloside from R. anthopogonoides [49]. Isomeric to $\mathbf{5 0}$, flavonol $\mathbf{4 9}$ is most likely myricetin-3-O-neohesperidoside found in Physalis angulata [50], while 47 is close to myricetin-3-O-rutinoside-7-O-glucoside from Limnanthes douglasii [51]. The myricetins with hexose/desoxyhexose ratio 2:2-3:3 remain unknown.

Acidic myricetin glycosides have fragments of hexuronic acid and hexose in ratios of 1:0 (myricetin $O$-hexuronide, 99), 1:1 (myricetin $O$-hexoside- $O$-hexuronide, 97), 1:2 (myricetin $O$-hexoside-di-O-hexuronide, 94), 2:1 (myricetin di-O-hexoside-O-hexuronide, 93), or 2:2 (myricetin di-O-hexoside-di-O-hexuronide; 91, 92). We were able to find information detailing one myricetin-3-O-glucuronide (the analog of 99) described in many plant families, among them the ericaceous genus Richea [61] although none in Rhododendron. Acylated myricetins contained both neutral and acidic carbohydrates, such as $O$-hexoside- $O$-acetates $(107,111,118,119), O$-hexoside- $O$-gallates $(165-169)$, and $O$-hexouronide- $O$-acetates $(106,108)$, while none of them have analogs or close structure among the known phytochemicals.

The identified quercetin glycosides were rutin (53), hyperoside (54), isoquercitrin (55), avicularin (57), quercitrin (59), miquelianin (15), and quercetin-3-O-(6' '-O-galloyl)glucoside (170). Compounds 53, 57, and 59 are known flavonoids of R. adamsii [12,13], while 54 and 55 have been reported in many rhododendrons [49]. The unknown non-acylated quercetins were $O$-hexoside- $O$-desoxyhexosides $(38,48)$ and $O$-hexoside-O-hexuronides $(\mathbf{1 0 0}$, $101,103,104)$, while acyl-fragments gave $O$-hexoside- $O$-acetates $(121,122), O$-hexuronide$O$-acetates $(\mathbf{1 1 0}, \mathbf{1 1 4}, \mathbf{1 1 5})$, and $O$-hexoside-O-gallate (171). Among kaempferols, we detected only two derivatives, juglanin (60) and afzelin (61), both previously described in rhododendrons [49]. Free myricetin (65), quercetin (66), and kaempferol (73) have previously been isolated from $R$. adamsii leaves [12,13], while isorhamnetin (69) was found for the first time here.

Dihydroflavonols found in R. adamsii were derivatives of dihydromyricetin (ampelopsin), dihydroquercetin (taxifolin), and dihydrokaempferol (aromadendrin). Dihydromyricetin detected in leaves of $R$. decorum and R. mucronulatum [49] was found as two glycosides, di-O-hexoside (39) and $O$-hexoside (40), both with no analogs in plants. Only dihydromyricetin $O$-pentoside has been previously characterized as a component of $R$. ferrugineum [51]. Free dihydroquercetin (taxifolin, 62) is a known flavonoid of $R$. adamsii [12], unlike nine of its glycosides, which were $O$-hexoside (44), 3-O-rhamnoside (astilbin, 45), O-hexuronide (102), n-O-hexoside-n-O-desoxyhexosides (41-43), and O-hexuroniden-O-acetates $(109,112,113)$. Taxifolin glycosides have been isolated from various rhododendrons, including 3-O-xyloside and 3-O-rhamnoside from $\mathrm{R}$. spinuliferum and 3-Oarabinoside from R. mucronulatum and R. ferrugineum [49]. Hexosides and desoxyhexosylhexosides of dihydroquercetin are newly demonstrated in the Rhododendron genus. Free dihydrokaempferol (aromadendrin, 64) has previously been described in R. decorum [49], although this is the first time it has been identified in $R$. adamsii.

The trace flavonoid group of R. adamsii included flavones identified as luteolin (67), apigenin (68), and farrerol (74) after comparison with reference standards. Farrerol has previously been described in R. adamsii [13] and R. dauricum [49].

\subsubsection{Prenylated Phenols}

A rare group of Rhododendron metabolites, prenylated phenols or phytocannabinoids, that were found in $R$. adamsii include three structural type-like derivatives of cannabigerorcinic acid $(\mathbf{7 0}-\mathbf{7 2}, 75-83,116,117,120,123,124,129)$, grifolic acid (125-128, 130-132, 137-140), and daurichromenic acid (133-136, 141-145) [62].

Cannabigerorcinic acid (120), or 2,4-dihydroxy-6-methyl-3-[(2E)-3,7-dimethyl-2,6octadien-1-yl]-benzoic acid, was identified using the reference standard showing a specific UV pattern $\left(\lambda_{\max } 220,268,306 \mathrm{~nm}\right)$ (Figure 6). Mass spectra demonstrated loss of water $(\mathrm{m} / z 303 \rightarrow 285), \mathrm{CO}(\mathrm{m} / z 303 \rightarrow 275)$, and fragments of $\mathrm{C}_{5} \mathrm{H}_{9}(\mathrm{~m} / z 303 \rightarrow 234)$ and $\mathrm{C}_{10} \mathrm{H}_{17}$ 
$(\mathrm{m} / \mathrm{z} 303 \rightarrow 166)$, which are characteristic for the orcinoids and cannabigerol type of phytocannabinoids [53]. Compound $\mathbf{1 2 0}$ is a typical component of Cannabis plants [63] and has previously been found in the $\mathrm{CO}_{2}$ extract of $R$. adamsii [13]. Two mono-O-acetates, 123 and 124, and one di-O-acetate 129 of cannabigerorcinic acid showed a typical loss of one and two acetyl fragments, respectively, with mass 42 a.m.u. More polar derivatives of $\mathbf{1 2 0}$ were identified as di-O-hexoside (116) and mono-O-hexoside (117), due to the registered loss of hexosyl residues with a mass 162 a.m.u. The known analogs of cannabigerorcinic acid acetates and glycosides are still undiscovered.


Figure 6. Mass spectra (A,B); negative ionization), UV patterns (C) of cannabigerorcinic acid (CGA) and cannabigerorcinic acid methyl ester (CGA-Me), and fragmentation way of CGA-Me (D).

Compound 79, which dominated in the high retention time fragment of the chromatogram of SPE-2 eluate, showed a UV pattern close to 120, however, its deprotonated ion was 14 a.m.u. higher with $m / z 317$ (Figure 6). The easy loss of a 14 a.m.u. fragment indicated this compound was a methyl ester of $\mathbf{1 2 0}(\mathrm{m} / z 317 \rightarrow 303)$, a known component of $R$. adamsii [11]. The isomeric to $\mathbf{7 9}$ mono-methyl esters $\mathbf{8 0}$, two dimethyl esters $\mathbf{8 1}$ and 82, and trimethyl ester $\mathbf{8 3}$ were found in $R$. adamsii for the first time, as were glycosides of cannabigerorcinic acid methyl ester, including di-O-hexosides $(\mathbf{7 0}, \mathbf{7 1}), \mathrm{O}$-hexoside- $\mathrm{O}$ desoxyhexoside (72), and $O$-hexoside (75), and three $O$-hexosides of cannabigerorcinic acid di-O-methyl ester (76-78).

Grifolic acid (137), or 2,4-dihydroxy-6-methyl-3-[(2E,6E)-3,7,11-trimethyl-2,6,10-dodecatrien1-yl]-benzoic acid, was identified by comparison with a reference standard. Grifolic acid demonstrated a UV pattern close to $\mathbf{1 2 0}$ and mass spectra indicating the presence of a longer prenyl fragment in the side chain (Figure 7); this compound has previously been detected in Rhododendron dauricum [64], although not in R. adamsii. Some esters of grifolic acid were also found in the plant as mono- $O$-methyl ester 138, di-O-methyl ester 139, and $O$-methyl ester- $O$-acetate 140, as well di-O-hexoside 131 and mono- $O$-hexoside 132, all of which remain unknown. 



Figure 7. Mass spectra (A,B); negative ionization), UV patterns (C) of grifolic acid (GRA) and hydroxy-grifolic acid (HGRA), and fragmentation way of GRA (D).

Compound 130 has a lower retention time than $137\left(t_{R} 10.67\right.$ vs. $\left.11.64 \mathrm{~min}\right)$ and a 16 a.m.u. higher mass of the deprotonated ion ( $m / z 387$ vs. 371$)$ while the general mass spectral pattern is similar, indicating an additional hydroxyl functional group in molecule 130 (Figure 7). The difference in the UV spectrum of hydroxy-grifolic acid was that it demonstrates a shoulder-like maxima at $270 \mathrm{~nm}$, in contrast to the true extreme curve of grifolic acid. The hydroxy-grifolic acid derivatives were four unknown glycosides, di-O-hexoside 125, mono-O-hexosides 126 and 127, and mono-O-pentoside 128.

Compound 141 was identified as daurichromenic acid, or 2-[(3E)-4,8-dimethyl-3,7nonadien-1-yl]-5-hydroxy-2,7-dimethylchromene-6-carboxylic acid, due to its specific UV and mass spectral patterns [54], which were matched with a reference standard (Figure 8). Four esters of daurichromenic acid were described as $O$-acetate (142), $O$-methyl ester (143), $O$-methyl ester- $O$-acetate (144), and di-O-methyl ester (145), while two glycosides were di-O-hexoside (133) and mono-O-hexoside (134).
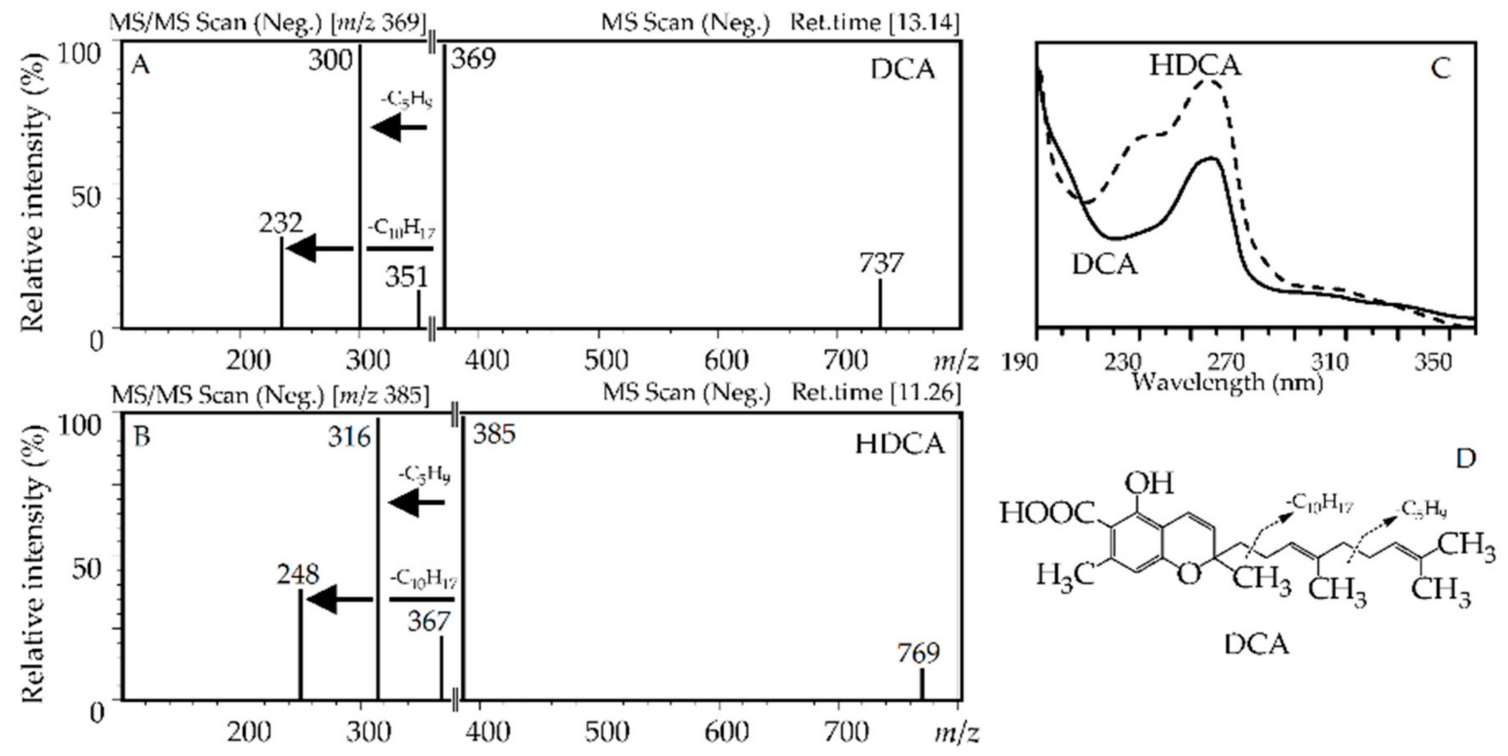

Figure 8. Mass spectra (A,B); negative ionization), UV patterns (C) of daurichromenic acid (DCA) and hydroxydaurichromenic acid (HDCA), and fragmentation way of DCA (D). 
The $m / z$ value of the deprotonated ion of compound 135 was 16 a.m.u. higher than that of daurichromenic acid, although the way of fragmentation was close to 141 (Figure 8). It was obvious that $\mathbf{1 3 5}$ was a hydroxy-derivative of daurichromenic acid with the additional hydroxyl group in the chromene fragment of the molecule, as indicated by the extra shortwave band in the UV spectrum. Additionally, the O-methyl ester of hydroxydaurichromenic acid (136) was found in R. adamsii. All derivatives of daurichromenic acid and hydroxy-daurichromenic acid have not previously been described.

Finally, thirty-eight prenylated phenols were found in $R$. adamsii, with only three of these compounds (cannabigerorcinic acid, cannabigerorcinic acid methyl ester, and daurichromenic acid) being previously described in the plant. Existing data regarding the bioactivity of Rhododendron cannabinoids demonstrate anti-HIV, antiallergic, anti-inflammatory, antithrombotic, antipsychotic, anticancer, and anti-Alzheimer potential [62,65], indicating a need for further study of $R$. adamsii phytocannabinoids.

\subsubsection{Benzoic Acid Derivatives, Hydroxycinnamates, and Dihydrochalcones}

Twenty benzoic acids of $R$. adamsii were derivatives of protocatechuic acid $(\mathbf{8 4}, \mathbf{8 5})$, vanillic/isovanillic acid (87-90), and gallic acid (146-151), including three compounds, vanillic acid 4-O-glucoside (89), gallic acid (148), and gallic acid $O$-methyl ester (151), that were identified using reference standards. Protocatechuic acid was found as di-O-hexoside (84) and mono-O-hexoside (85), vanillic/isovanillic acid as mono- $O$-hexosides $(\mathbf{8 7}, \mathbf{8 8}, \mathbf{9 0})$, gallic acid as di-O-hexoside (146) and mono- $O$-hexoside (147), and gallic acid $O$-methyl ester as mono-O-hexosides $(\mathbf{1 5 0 , 1 5 1 )}$. Previously, compounds 148 and 151 have been found in Rhododendron plants [16,49], however, benzoic acid O-hexosides are still unknown in the genus.

Four hydroxycinnamates, as mono-caffeoylquinic acids, were detected in $R$. adamsii and identified with 1-O- (86), 3-O- (96), 4-O- (98), and 5-O-caffeoylquinic acid (95) after comparison with reference standards. Chlorogenic acid is the only compound previously identified in R. kotschyi and R. mucronulatum [49].

Only one dihydrochalcone phloretin (63) was found in the methanol eluates (SPE-2) of $R$. adamsii leaves. A previous case of the discovery of $\mathbf{6 3}$ in Rhododendron genus refers to R. molle [49].

\subsubsection{Catechins and Procyanidins}

Eight catechins and five procyanidins were detected in R. adamsii leaves, including the reference standard identified compounds catechin (155), epicatechin (159), catechin 3-O-gallate (161), epicatechin 3-O-gallate (164), and procyanidins $B_{1}(154), B_{2}(158)$ and $\mathrm{C}_{1}$ (160). Additional compounds were catechin/epicatechin di-O-hexoside (152) and mono-O-hexosides $(\mathbf{1 5 3}, \mathbf{1 5 7})$, catechin/epicatechin $O$-gallate-O-hexoside $(\mathbf{1 5 6})$, and catechin/epicatechin dimer $O$-gallate (162) and di-O-gallate (163). Catechins and procyanidins are usual components of both Rhododendron species and Ericaceae plants [49], with catechin/epicatechin $O$-hexosides referred to in genus for the first time.

\subsubsection{Chemotaxonomic Significance of R. adamsii Metabolites}

The tribe Fragrantica E. Busch. The Rhododendron genus includes the Siberian species $R$. adamsii as well as the Himalayan species R. anthopogon D. Don, Chinese species R. anthopogonoides Maxim. and R. cephalanthum Franch., and Afghan species R. collettianum Aitch. \& Hemsl. [66]. Despite the varying levels of knowledge of Fragrantica species chemistry, we found information regarding ursolic acid [67], epicatechin, hyperoside, and quercitrin [68] in R. anthopogon; quercetin, isorhamnetin, hyperoside [69], kaempferol, taxifolin, ursolic acid [70], and phytocannabinoids [71,72] in R. anthopogonoides; and quercetin [73] and phytocannabinoids [74] in R. collettianum. Flavonols of the quercetin and kaempferol type, dihydroflavonols (taxifolin), and ursolic acid have a wide distribution in the Rhododendron genus [49], while phytocannabinoids of orcinoids and the cannabigerol type are still rare Ericaceous compounds and could be specific markers of Fragrantica series rhododendrons. 


\subsubsection{Seasonal Variation of $R$. adamsii Metabolites}

Non-deciduous (or evergreen) plants, such as R. adamsii, can protect the integrity of their leaves, irrespective of environmental temperatures and season. Due to this feature, people collect the leaves of $R$. adamsii year-round, however, there is no information about the seasonal variation of metabolites. Quantification data of 171 compounds in 215 samples of $R$. adamsii leaves, collected across four seasons, demonstrated significant variability in the contents of all non-trace compounds (Table 3). Grouping the compounds into two clusters (phenolics and non-phenolics), it can be seen that the content of both clusters increased from January to July and reduced in December (Table 4). The maximal phenolics/nonphenolics level was in July $(186.28 / 131.47 \mathrm{mg} / \mathrm{g})$ while the lowest level was in January and March $(97.26 / 48.95 \mathrm{mg} / \mathrm{g})$. This means that more extractable compounds accumulate in summer samples.

Table 4. Seasonal variation of the total content of compound groups in R. adamsii leaves, $\mathrm{mg} / \mathrm{g}$ of dry plant weight.

\begin{tabular}{|c|c|c|c|c|c|c|c|c|}
\hline \multirow{2}{*}{ Group of Compounds } & \multicolumn{8}{|c|}{ Content, mg/g } \\
\hline & January & March & May & June & July & August & October & December \\
\hline Total carbohydrates & 44.25 & 44.85 & 38.40 & 74.79 & 91.82 & 63.21 & 63.99 & 54.48 \\
\hline Total organic acids & 1.02 & 9.21 & 23.03 & 31.17 & 20.90 & 10.98 & 3.11 & 2.21 \\
\hline Total simple phenol glycosides & 6.94 & 8.23 & 12.40 & 15.41 & 19.45 & 20.36 & 11.33 & 10.28 \\
\hline incl. phloroglucinol derivatives & 3.48 & 4.45 & 6.91 & 8.66 & 11.73 & 13.33 & 5.16 & 4.62 \\
\hline incl. hydroquinone derivatives & 0.32 & 0.32 & 0.76 & 0.82 & 0.93 & 0.65 & 0.41 & 0.35 \\
\hline incl. orcinol derivatives & 3.14 & 3.46 & 4.73 & 5.93 & 6.79 & 6.38 & 5.76 & 5.31 \\
\hline Total triterpene glycosides & 3.68 & 5.88 & 12.00 & 15.90 & 18.75 & 19.22 & 14.98 & 4.10 \\
\hline Total flavonols & 18.47 & 16.98 & 47.54 & 58.81 & 71.69 & 61.54 & 45.19 & 26.34 \\
\hline $\begin{array}{l}\text { incl. glycosides, myricetin } \\
\text { derivatives }\end{array}$ & 15.61 & 14.47 & 41.75 & 50.86 & 60.52 & 52.56 & 39.37 & 21.43 \\
\hline $\begin{array}{c}\text { incl. glycosides, quercetin } \\
\text { derivatives }\end{array}$ & 1.04 & 1.37 & 5.45 & 7.61 & 10.89 & 8.63 & 3.71 & 2.37 \\
\hline $\begin{array}{c}\text { incl. glycosides, kaempferol } \\
\text { derivatives }\end{array}$ & $<0.01$ & $<0.01$ & $<0.01$ & $<0.01$ & $<0.01$ & $<0.01$ & $<0.01$ & $<0.01$ \\
\hline incl. aglycones & 1.82 & 1.14 & 0.51 & 0.34 & 0.28 & 0.35 & 1.81 & 2.54 \\
\hline Total dihydroflavonols & 8.31 & 8.33 & 8.29 & 9.64 & 10.95 & 10.12 & 8.94 & 9.26 \\
\hline $\begin{array}{c}\text { incl. dihydromyricetin } \\
\text { derivatives }\end{array}$ & 1.53 & 1.48 & 2.73 & 3.11 & 3.52 & 3.18 & 2.39 & 2.01 \\
\hline $\begin{array}{c}\text { incl. dihydroquercetin } \\
\text { derivatives }\end{array}$ & 6.67 & 6.85 & 5.56 & 6.53 & 7.43 & 6.94 & 6.55 & 7.11 \\
\hline $\begin{array}{c}\text { incl. dihydrokaempferol } \\
\text { derivatives }\end{array}$ & 0.11 & $<0.01$ & $<0.01$ & $<0.01$ & $<0.01$ & $<0.01$ & $<0.01$ & 0.14 \\
\hline Total flavones & 0.52 & $<0.01$ & $<0.01$ & $<0.01$ & $<0.01$ & $<0.01$ & 0.62 & 1.58 \\
\hline Total prenylated phenols & 58.87 & 51.67 & 34.21 & 38.49 & 41.60 & 54.42 & 67.69 & 71.13 \\
\hline $\begin{array}{c}\text { incl. cannabigerorcinic acid } \\
\text { derivatives }\end{array}$ & 39.63 & 34.90 & 24.57 & 27.31 & 25.18 & 31.96 & 43.59 & 47.14 \\
\hline incl. grifolic acid derivatives & 11.74 & 10.40 & 8.35 & 9.14 & 13.46 & 17.46 & 17.53 & 15.95 \\
\hline $\begin{array}{c}\text { incl. daurichromenic acid } \\
\text { derivatives }\end{array}$ & 7.50 & 6.37 & 1.29 & 2.04 & 2.96 & 5.00 & 6.57 & 8.04 \\
\hline Total benzoic acid derivatives & 0.26 & $<0.01$ & 2.92 & 6.30 & 9.75 & 8.73 & 4.63 & 1.42 \\
\hline Total hydroxycinnamates & $<0.01$ & $<0.01$ & 0.70 & 0.82 & 0.97 & 0.52 & 0.30 & 0.08 \\
\hline Total catechins & 9.51 & 7.69 & 10.84 & 12.56 & 19.02 & 19.21 & 14.53 & 11.17 \\
\hline Total procyanidins & 1.67 & 1.36 & 2.13 & 3.33 & 4.26 & 4.00 & 2.41 & 2.05 \\
\hline Total dihydrochalcones & 0.18 & $<0.01$ & $<0.01$ & $<0.01$ & $<0.01$ & $<0.01$ & 0.23 & 0.29 \\
\hline Total phenolics & 107.66 & 97.26 & 126.04 & 153.03 & 186.28 & 184.97 & 159.70 & 136.77 \\
\hline Total non-phenolics & 48.95 & 59.94 & 73.43 & 121.86 & 131.47 & 93.41 & 82.08 & 60.79 \\
\hline Total phenolics/non-phenolics & 156.61 & 157.20 & 199.47 & 274.89 & 317.75 & 278.38 & 241.78 & 197.56 \\
\hline
\end{tabular}

The content levels of the smaller groups of compounds conform to the same rule, with the exception of prenylated phenols. Increased levels of grifolic acid derivatives were observed in October samples, with values up to $17.53 \mathrm{mg} / \mathrm{g}$ vs. $8.35 \mathrm{mg} / \mathrm{g}$ in 
May samples, while cannabigerorcinic acid derivatives in the December samples reached $47.14 \mathrm{mg} / \mathrm{g}$ vs. $25.18 \mathrm{mg} / \mathrm{g}$ in July samples and daurichromenic acid derivatives in December reached $8.04 \mathrm{mg} / \mathrm{g}$ vs. $1.29 \mathrm{mg} / \mathrm{g}$ in May samples. Close variations in content were found for the flavonoid aglycones that showed the highest amounts in December samples, such as flavonol aglycones $(2.54 \mathrm{mg} / \mathrm{g}$ in December vs. $0.28 \mathrm{mg} / \mathrm{g}$ in July) and flavone aglycones $(1.58 \mathrm{mg} / \mathrm{g}$ in December vs. trace content in the spring and summer). Lipophilic compounds differed from the core metabolites with medium and high polarity in a seasonal variation pattern.

The flavonoid aglycones are the usual components of leaf surface wax, which accumulates in the winter period and plays an ecophysiological function in plant development $[75,76]$, unlike the little-known prenylated phenols of rhododendrons. In this regard, we assumed that the prenylated phenols of $R$. adamsii are the leaves' surface components, which was confirmed after the analysis of surface diethyl ether extract. Derivatives of cannabigerorcinic acid, grifolic acid, and daurichromenic acid were detected in ether extract at a high level, whilst trace content was detected in the extract of ether-treated leaves (Figure S3). The total yield of ether extract was at a maximal in December samples (25.3\% of dry leaf weight) and a minimal in July samples (11.24\% of dry leaf weight) (Figure S4); this enables us to suggest that lipophilic prenylated phenols of $R$. adamsii have a protective effect in the winter period on an equal basis with other lipids covered on the leaf surface of evergreen plants [77-79].

The results of principal component analysis (PCA) of the content of 171 compounds in 215 samples of $R$. adamsii leaves, collected in various months of the year, showed that the specific distribution of individual points on the diagram, all located on the sides of the round that means the metabolic changes occur gradually (Figure 9).

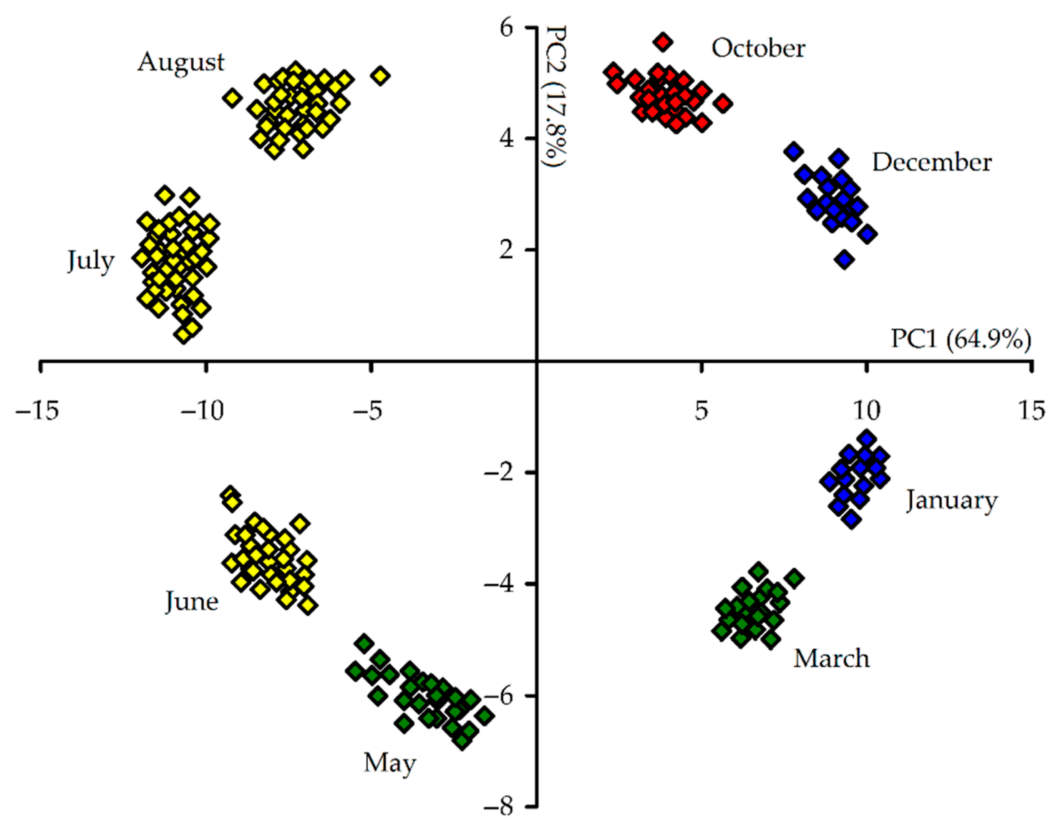

Figure 9. Principal component analysis (PCA) for the content of 171 compounds in 215 samples of $R$. adamsii leaves collected during various months of the year.

The chemical study undertaken has demonstrated that the extractable metabolites of $R$. adamsii leaves are a very complex mixture of compounds of different nature and polarity, with significant variation depending on the season of collection. We expected to discover variable bioactivity in $R$. adamsii extracts prepared from plant materials collected during different seasons, an idea that was confirmed by our subsequent experiments. 


\subsection{Bioactivity of R. adamsii Extracts: Seasonal Changes of Antioxidant and Adaptogenic Potential}

\subsubsection{Antioxidant Activity}

Seasonal variation of the $R$. adamsii metabolome reflected varying contents of the bioactive compounds, as expected. Extracts prepared from plant material collected in four different seasons (January, May, July, and October) was inspected for antioxidant potential via nine traditional assays [80], including radical scavenging against 2,2-diphenyl1-picrylhydrazyl radicals ( $\left.\mathrm{DPPH}^{\bullet}\right), 2,2^{\prime}$-azino-bis(3-ethylbenzothiazoline-6-sulfonic acid) radicals $\left(\mathrm{ABTS}^{\bullet+}\right), \mathrm{N}, \mathrm{N}$-dimethyl-p-phenylenediamine radicals $\left(\mathrm{DMPD}^{\bullet+}\right)$, superoxide radicals $\left(\mathrm{O}_{2}{ }^{\bullet-}\right)$, hydroxyl radicals $\left({ }^{\bullet} \mathrm{OH}\right)$, and chlorine radicals $\left(\mathrm{Cl}^{\bullet}\right)$, as well as carotene bleaching assay, nitric oxide scavenging assay, and ferrous ions $\left(\mathrm{Fe}^{2+}\right)$ chelating ability (Table 5). Ten selected compounds that are representatives of various metabolite groups found in R. adamsii were also analyzed.

Table 5. Antioxidant activity of R. adamsii extracts and selected compounds.

\begin{tabular}{|c|c|c|c|c|c|c|c|c|c|}
\hline $\begin{array}{l}\text { Extract (Collection } \\
\text { Month), Compound }\end{array}$ & $\mathrm{DPPH}^{\bullet b}$ & $\mathrm{ABTS}^{\bullet+b}$ & $\mathrm{DMPD}^{\bullet+b}$ & $\mathrm{O}_{2}^{\bullet-b}$ & $\mathrm{OH}^{\bullet} \mathrm{b}$ & $\mathrm{CBA}^{\mathrm{b}}$ & $\mathrm{Cl}^{\bullet \mathrm{c}}$ & $\mathrm{NO}^{\mathrm{d}}$ & $\mathrm{FeCA}^{\mathrm{e}}$ \\
\hline $\begin{array}{l}\text { R. adamsii extract } \\
\text { (January) }\end{array}$ & $\begin{array}{c}25.37 \pm \\
0.68\end{array}$ & $\begin{array}{c}15.80 \pm \\
0.31\end{array}$ & $\begin{array}{c}87.35 \pm \\
2.53\end{array}$ & $\begin{array}{c}82.11 \pm \\
2.46\end{array}$ & $\begin{array}{c}59.73 \pm \\
1.79\end{array}$ & $\begin{array}{c}30.62 \pm \\
1.29\end{array}$ & $\begin{array}{l}263.93 \\
\pm 6.55\end{array}$ & $<5$ & $\begin{array}{c}129.03 \pm \\
5.12\end{array}$ \\
\hline $\begin{array}{l}\text { R. adamsii extract } \\
\text { (May) }\end{array}$ & $\begin{array}{c}9.82 \pm \\
0.21\end{array}$ & $\begin{array}{c}10.54 \pm \\
0.21\end{array}$ & $\begin{array}{c}53.62 \pm \\
1.61\end{array}$ & $\begin{array}{c}52.69 \pm \\
1.54\end{array}$ & $\begin{array}{c}15.25 \pm \\
0.42\end{array}$ & $\begin{array}{c}15.35 \pm \\
0.61\end{array}$ & $\begin{array}{c}408.34 \\
\pm \\
10.26\end{array}$ & $\begin{array}{c}4.05 \pm \\
0.19\end{array}$ & $\begin{array}{c}193.55 \pm \\
7.63\end{array}$ \\
\hline $\begin{array}{l}\text { R. adamsii extract } \\
\text { (July) }\end{array}$ & $\begin{array}{c}3.27 \pm \\
0.06\end{array}$ & $\begin{array}{c}8.25 \pm \\
0.16\end{array}$ & $\begin{array}{c}37.53 \pm \\
1.12\end{array}$ & $\begin{array}{c}25.83 \pm \\
0.77\end{array}$ & $\begin{array}{c}5.43 \pm \\
0.16\end{array}$ & $\begin{array}{c}12.50 \pm \\
0.53\end{array}$ & $\begin{array}{c}475.62 \\
\pm \\
11.89\end{array}$ & $\begin{array}{c}3.67 \pm \\
0.16\end{array}$ & $\begin{array}{c}211.74 \pm \\
8.44\end{array}$ \\
\hline $\begin{array}{l}\text { R. adamsii extract } \\
\text { (October) }\end{array}$ & $\begin{array}{c}12.62 \pm \\
0.31 \\
>100\end{array}$ & $\begin{array}{c}12.32 \pm \\
0.24 \\
>100\end{array}$ & $\begin{array}{c}63.82 \pm \\
1.99 \\
>100\end{array}$ & $\begin{array}{l}49.63 \pm \\
1.45 \\
>200\end{array}$ & $\begin{array}{c}26.82 \pm \\
0.73 \\
>100\end{array}$ & $\begin{array}{c}27.09 \pm \\
1.08 \\
>200\end{array}$ & $\begin{array}{l}378.21 \\
\pm 9.40 \\
<1\end{array}$ & $\begin{array}{c}4.89 \pm \\
0.22 \\
<5\end{array}$ & $\begin{array}{c}173.62 \pm \\
6.90 \\
<1\end{array}$ \\
\hline Phlorin & $\begin{array}{c}52.06 \pm \\
1.63\end{array}$ & $>100$ & $>100$ & $>200$ & $>100$ & $>200$ & $\begin{array}{c}4.27 \pm \\
0.08\end{array}$ & $<5$ & $<1$ \\
\hline Ursolic acid & $>100$ & $>100$ & $>100$ & $>200$ & $>100$ & $>200$ & $<1$ & $<5$ & $<1$ \\
\hline $\begin{array}{l}\text { Myricetin-3-O- } \\
\text { glucoside }\end{array}$ & $\begin{array}{c}5.83 \pm \\
0.12\end{array}$ & $\begin{array}{c}2.35 \pm \\
0.04\end{array}$ & $\begin{array}{c}18.89 \pm \\
0.56\end{array}$ & $\begin{array}{c}22.17 \pm \\
0.66\end{array}$ & $\begin{array}{c}3.81 \pm \\
0.10\end{array}$ & $\begin{array}{c}12.27 \pm \\
0.47\end{array}$ & $\begin{array}{c}893.57 \\
\pm \\
17.85\end{array}$ & $\begin{array}{c}1.07 \pm \\
0.04\end{array}$ & $\begin{array}{c}70.52 \pm \\
2.11\end{array}$ \\
\hline $\begin{array}{l}\text { Quercetin-3-O- } \\
\text { glucoside }\end{array}$ & $\begin{array}{c}9.36 \pm \\
0.18\end{array}$ & $\begin{array}{c}5.72 \pm \\
0.11\end{array}$ & $\begin{array}{c}62.65 \pm \\
1.86\end{array}$ & $\begin{array}{c}73.62 \pm \\
2.21\end{array}$ & $\begin{array}{c}12.63 \pm \\
0.39\end{array}$ & $\begin{array}{c}35.64 \pm \\
1.40\end{array}$ & $\begin{array}{c}569.21 \\
\pm \\
11.38\end{array}$ & $\begin{array}{c}2.35 \pm \\
0.09\end{array}$ & $\begin{array}{c}62.04 \pm \\
1.82\end{array}$ \\
\hline $\begin{array}{l}\text { Cannabigerorcinic } \\
\text { acid }\end{array}$ & $>100$ & $>100$ & $>100$ & $>200$ & $>100$ & $\begin{array}{c}89.63 \pm \\
3.59\end{array}$ & $\begin{array}{l}25.63 \\
\pm 0.50\end{array}$ & $<5$ & $\begin{array}{c}23.12 \pm \\
0.69\end{array}$ \\
\hline Grifolic acid & $>100$ & $>100$ & $>100$ & $>200$ & $>100$ & $\begin{array}{l}124.18 \\
\pm 4.96\end{array}$ & $\begin{array}{c}18.04 \\
\pm 0.32\end{array}$ & $<5$ & $\begin{array}{c}15.60 \pm \\
0.41\end{array}$ \\
\hline Daurichromenic acid & $>100$ & $>100$ & $>100$ & $>200$ & $>100$ & $\begin{array}{c}93.52 \pm \\
3.74\end{array}$ & $\begin{array}{l}22.57 \\
\pm 0.45\end{array}$ & $<5$ & $\begin{array}{c}12.09 \pm \\
0.34\end{array}$ \\
\hline Gallic acid & $\begin{array}{c}1.53 \pm \\
0.03\end{array}$ & $\begin{array}{c}0.86 \pm \\
0.02\end{array}$ & $\begin{array}{c}22.45 \pm \\
0.67\end{array}$ & $\begin{array}{c}20.14 \pm \\
0.58\end{array}$ & $\begin{array}{c}9.57 \pm \\
0.29\end{array}$ & $\begin{array}{c}5.92 \pm \\
0.23\end{array}$ & $\begin{array}{c}1267.02 \\
\pm \\
25.27\end{array}$ & $\begin{array}{c}0.97 \pm \\
0.03\end{array}$ & $\begin{array}{c}157.12 \pm \\
4.83\end{array}$ \\
\hline Catechin & $\begin{array}{c}3.02 \pm \\
0.06\end{array}$ & $\begin{array}{c}1.41 \pm \\
0.03\end{array}$ & $\begin{array}{c}20.39 \pm \\
0.60\end{array}$ & $\begin{array}{c}43.10 \pm \\
1.25\end{array}$ & $\begin{array}{c}7.73 \pm \\
0.23\end{array}$ & $\begin{array}{c}26.84 \pm \\
1.07\end{array}$ & $\begin{array}{c}853.14 \\
\pm \\
17.06\end{array}$ & $\begin{array}{c}1.56 \pm \\
0.06\end{array}$ & $\begin{array}{c}75.14 \pm \\
2.20\end{array}$ \\
\hline Trolox ${ }^{a}$ & $\begin{array}{c}8.89 \pm \\
0.15\end{array}$ & $\begin{array}{c}3.02 \pm \\
0.06\end{array}$ & $\begin{array}{l}53.10 \pm \\
1.59\end{array}$ & $\begin{array}{c}90.63 \pm \\
2.40\end{array}$ & $\begin{array}{c}10.25 \pm \\
0.26\end{array}$ & $\begin{array}{c}20.63 \pm \\
0.82\end{array}$ & 1000 & $\begin{array}{c}0.83 \pm \\
0.03\end{array}$ & $\begin{array}{c}42.72 \pm \\
1.26\end{array}$ \\
\hline
\end{tabular}

${ }^{a}$ Reference compound; ${ }^{\mathrm{b}} \mathrm{IC}_{50}, \mu \mathrm{g} / \mathrm{mL}{ }^{\mathrm{c}} \mathrm{mg}$ Trolox eq. $/ \mathrm{g} ;{ }^{\mathrm{d}} \mathrm{IC}_{50}, \mathrm{mg} / \mathrm{mL} ;{ }^{\mathrm{e}} \mathrm{mg} \mathrm{Fe}{ }^{2+} / \mathrm{g}$.

Study of the antioxidant potential of $R$. adamsii extracts against artificial radicals, such as $\mathrm{DPPH}^{\bullet}, \mathrm{ABTS}^{\bullet+}$, and $\mathrm{DMPD}^{\bullet+}$, demonstrated superior scavenging effects in July samples, with $\mathrm{IC}_{50}$ values of $3.27,8.25$, and $37.53 \mu \mathrm{g} / \mathrm{mL}$, respectively. Samples collected in January were less active, with $\mathrm{IC}_{50}$ values of $25.37,15.80$, and $87.35 \mu \mathrm{g} / \mathrm{mL}$ for $\mathrm{DPPH}^{\bullet}$, 
$\mathrm{ABTS}^{\bullet+}$, and $\mathrm{DMPD}^{\bullet+}$ radicals, respectively; the same parameters for Trolox, which was used as a reference compound, were $8.89,3.02$, and $53.10 \mu \mathrm{g} / \mathrm{mL}$, respectively. Oxygenradicals, as superoxide and hydroxyl radicals, were inactivated by all $R$. adamsii extracts, with the highest effectiveness observed for July samples $(25.83$ and $5.43 \mu \mathrm{g} / \mathrm{mL}$, respectively), which showed a good protective effect in the carotene bleaching assay $(12.50 \mu \mathrm{g} / \mathrm{mL}$ vs. $20.63 \mu \mathrm{g} / \mathrm{mL}$ for Trolox) and chlorine-radical scavenging assay $(475.62 \mu \mathrm{g} / \mathrm{mL}$ vs. $1000 \mu \mathrm{g} / \mathrm{mL}$ for Trolox).

The ability of $R$. adamsii extracts to scavenge nitric oxide molecules was poor $(3.67 \mathrm{mg} / \mathrm{mL}$ for July sample) in comparison to Trolox $(0.83 \mathrm{mg} / \mathrm{mL})$, in contrast to the $\mathrm{Fe}^{2+}$-chelating ability of $R$. adamsii extract, which reached a maximum in the July sample (211.74 $\mathrm{mg}$ $\mathrm{Fe}^{2+} / \mathrm{g}$ ) and minimum in the January sample (129.03 $\left.\mathrm{mg} \mathrm{Fe}{ }^{2+} / \mathrm{g}\right)$, still exceeding the Trolox value (42.72 $\mathrm{mg} \mathrm{Fe}^{2+} / \mathrm{g}$ ). The compounds providing the most significant impact on artificial/oxygen/chlorine/nitric oxide radical scavenging ability and $\mathrm{Fe}^{2+}$-chelating ability were gallic acid, catechin, myricetin-3-O-glucoside, and quercetin-3-O-glucoside, which are also known as radical-scavengers [81], unlike prenylated phenols, phenol glycosides, triterpenes, and organic acids, which were inactive or at low activity levels. Thus, the high seasonal content of flavonoids and catechins is the reason why July samples of $R$. adamsii extract are the most active in all antioxidant assays studied.

Previously, some fungal prenylated phenols have been concluded to be medium effectiveness antioxidants, with $\mathrm{IC}_{50}$ values in the $\mathrm{DPPH}$ assay ranging from $60-80 \mu \mathrm{g} / \mathrm{mL}$ [82]; therefore, our results are not that surprising. The HPLC-DAD assay coupled with precolumn incubation of $R$. adamsii extract with $\mathrm{DPPH}^{\bullet}$ solution showed an almost complete reduction of the flavonoid peaks area, indicating their primary activity in the free radical scavenging process (Figure 10).

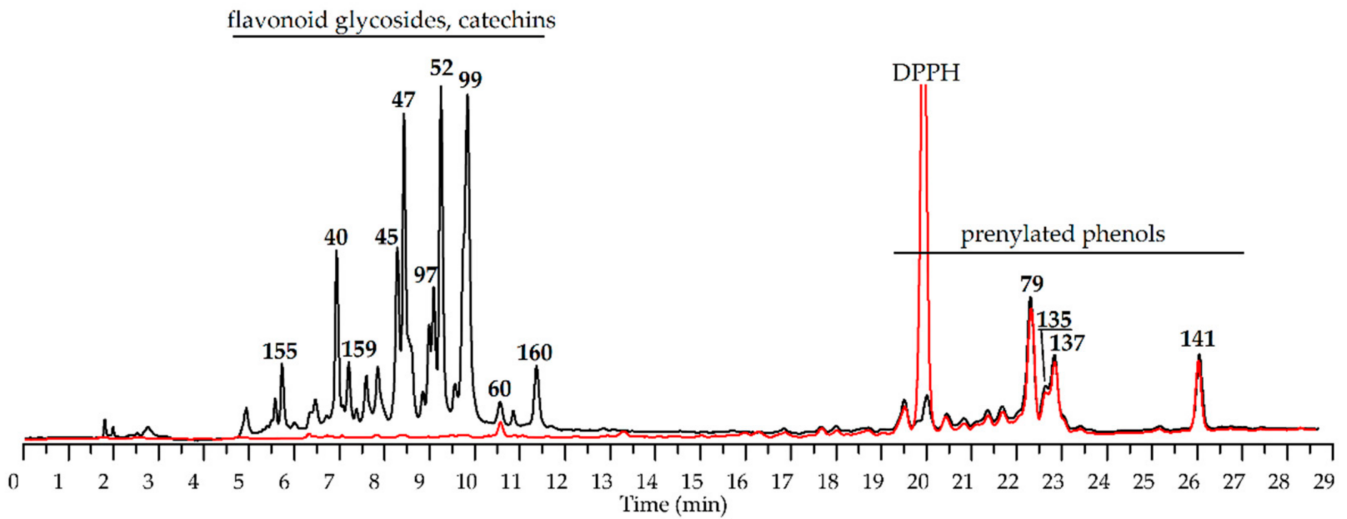

Figure 10. HPLC-UV chromatograms of R. adamsii leave total extract (July sample) before (black) and after preincubation (red) with $\mathrm{DPPH}^{\bullet}$ radicals solution. The excess of $\mathrm{DPPH}^{\bullet}$ radicals signed as DPPH. The basic peaks are numbered as described in Table 3.

Derivatives of cannabigerorcinic, grifolic, and daurichromenic acid did not demonstrate visible changes of peak area due to weak activity. There is a clear demonstration of the important role of selected phenolic compounds (flavonoids, catechins) in the antioxidant properties of R. adamsii extracts.

\subsubsection{Adaptogenic Activity}

In further examination of the adaptogenic potential of $R$. adamsii, we analyzed the effectiveness of four leaf extracts with different seasonal origins-January, May, July, and October, in a two-step to exhaustion swimming test assessing the influence of the remedy on physical performance (first step) and endurance (second step). Animals in the control group, which received saline, showed 16.5 and 3.9 min swimming times in the 1st and 2nd steps of the test, compared to the R. rosea extract group used as a positive control, which showed 37.4 and 18.8 min swimming times in the two steps, respectively (Figure 11). 
The observed differences in the results of the $R$. rosea group compared to the saline group demonstrate stimulation of physical performance and the endurance of the animals.



Figure 11. Effects of four different $R$. adamsii leaf extracts (January, May, July, and October samples) and $R$. rosea extract $(50 \mathrm{mg} / \mathrm{kg})$ on swimming time of mice in a two-step swimming to exhaustion test. ${ }^{*}-p<0.05$ vs. saline group.

Statistically significant $(p<0.05)$ increases in swimming time were observed in all experimental groups receiving $R$. adamsii leaf extracts when compared to the control group. The July group showed the best results, with values of 34.1 and $21.3 \mathrm{~min}$ in the first and second swimming steps, respectively, indicating that $R$. adamsii positively influences physical performance and endurance. The remaining $R$. adamsii groups demonstrated progressive decreases in swimming times when analyzing samples from the May to January groups, similar to the pattern observed for changes in the antioxidant potential of the extracts.

The two-step swimming to exhaustion test, as an exhaustive exercise, reflects on the biochemical parameters of the experimental animals. This exercise leads to fatigue and inability by the animals to perform any further active swimming movements, resulting from decreased macroergic compound content in the skeletal muscles, almost complete exhaustion of glucose concentrations in the blood and glycogen in the liver, and, accordingly, the accumulation of lactate and pyruvate [83]. Compared with intact mice, the saline group animals showed significant decreases in adenosine triphosphate (ATP; $359 \mathrm{vs} .71 \mathrm{pmol} / \mathrm{g}$ ) and creatine phosphate (CP; 3215 vs. $937 \mathrm{pmol} / \mathrm{g})$ in their skeletal muscles, as well as decreased glucose levels in the blood serum ( $9.5 \mathrm{vs.} 1.1 \mathrm{mmol} / \mathrm{L})$ and decreased glycogen content in the liver ( $23.3 \mathrm{vs} .5 .7 \mathrm{mg} / \mathrm{g}$ ), accompanied by increased levels of lactate in the skeletal muscle ( 3.7 vs. $8.8 \mu \mathrm{mol} / \mathrm{kg}$ ) and pyruvic acid in the blood serum ( $210 \mathrm{vs}$. $1408 \mathrm{pg} / \mathrm{mL}$ ) (Table 6). The expressed consumption of macroergic compounds (ATP, CP) and accumulation of acidic products in the animals points to very intense physical stress resulting in oxidative misbalance due to the accumulation of malondialdehyde (MDA; 1.8 vs. $6.7 \mathrm{nmol} / \mathrm{L})$ and decreases catalase activity in the blood serum (11.5 vs. $6.2 \mathrm{mcat} / \mathrm{L}$ ).

A different picture emerged following similar physical activity after intake of either $R$. adamsii or $R$. rosea extracts by the animals. Both extracts, in doses of $50 \mathrm{mg} / \mathrm{kg}$, resulted in increased working ability due to metabolic restructuring through an improvement in the energy supply of skeletal muscles, as evidenced by increases in ATP and CP contents equivalent to 1.7-2.4 times the values of the control group. Also, increased carbohydrate reserves were observed, as indicated by elevated levels of blood serum glucose and liver glycogen, while reduced levels of lactate, observed in the skeletal muscle, and pyruvate, in the blood serum, pointed to decreased levels of metabolic acidosis. The level of MDA in blood serum decreased, from $6.7 \mathrm{nmol} / \mathrm{L}$ in the control group to $2.8 \mathrm{nmol} / \mathrm{L}$ in the $R$. adamsii group and $2.3 \mathrm{nmol} / \mathrm{L}$ in the $R$. rosea group, while catalase activity levels were similar in these groups to the intact group value. Thus, under strong physical stress, $R$. adamsii demonstrated the most important properties of an adaptogenic drug, providing 
more economical use of the energy substrates and increasing the body's ability to function optimally with less energy consumption [84].

Table 6. Influence of R. adamsii leaf extract (July sample, dose $50 \mathrm{mg} / \mathrm{kg}$ ) and R. rosea extract (dose $50 \mathrm{mg} / \mathrm{kg}$ ) on biochemical parameters of the skeletal muscles, blood serum, and liver of mice after the two-step swimming test.

\begin{tabular}{|c|c|c|c|c|c|c|c|c|}
\hline \multirow[b]{2}{*}{$\begin{array}{c}\text { Experimental } \\
\text { Group }\end{array}$} & \multicolumn{3}{|c|}{ Skeletal Muscles } & \multicolumn{4}{|c|}{ Blood Serum } & \multirow{2}{*}{$\begin{array}{c}\text { Liver } \\
\begin{array}{c}\text { Glycogen, } \\
\text { mg/g }\end{array}\end{array}$} \\
\hline & $\begin{array}{c}\text { ATP, } \\
\mathrm{pmol} / \mathrm{g}\end{array}$ & $\begin{array}{c}\text { Creatine } \\
\text { Phosphate, } \\
\text { pmol/g }\end{array}$ & $\begin{array}{l}\text { Lactate, } \\
\mu \mathrm{mol} / \mathrm{kg}\end{array}$ & $\begin{array}{c}\text { Pyruvic } \\
\text { Acid, pg/mL }\end{array}$ & $\begin{array}{l}\text { Glucose, } \\
\mathrm{mmol} / \mathrm{L}\end{array}$ & $\begin{array}{l}\text { MDA, } \\
\text { nmol/L }\end{array}$ & $\begin{array}{l}\text { Catalase, } \\
\text { mcat } / \mathrm{L}\end{array}$ & \\
\hline $\begin{array}{l}\text { Saline, without } \\
\text { test (intact) }\end{array}$ & $359 \pm 71 *$ & $3215 \pm 160$ * & $3.7 \pm 0.2 *$ & $210 \pm 57^{*}$ & $9.5 \pm 1.5^{*}$ & $1.8 \pm 0.1 *$ & $11.5 \pm 0.7^{*}$ & $23.3 \pm 1.1^{*}$ \\
\hline $\begin{array}{l}\text { Saline, after test } \\
\text { (control) }\end{array}$ & $71 \pm 17$ & $937 \pm 53$ & $8.8 \pm 0.6$ & $1408 \pm 281$ & $1.1 \pm 0.2$ & $6.7 \pm 0.5$ & $6.2 \pm 0.5$ & $5.7 \pm 0.3$ \\
\hline $\begin{array}{c}\text { R. adamsii, } \\
\text { without test }\end{array}$ & $325 \pm 58 *$ & $3107 \pm 156$ * & $3.7 \pm 0.2 *$ & $215 \pm 55$ * & $9.7 \pm 1.7^{*}$ & $1.8 \pm 0.1$ * & $11.5 \pm 0.7$ * & $22.9 \pm 1.1$ * \\
\hline $\begin{array}{l}\text { R. adamsii, after } \\
\text { test }\end{array}$ & $143 \pm 44$ * & $1631 \pm 98 *$ & $5.2 \pm 0.3 *$ & $806 \pm 145 *$ & $3.3 \pm 0.5 *$ & $2.8 \pm 0.2 *$ & $10.0 \pm 0.8^{*}$ & $12.8 \pm 0.8 *$ \\
\hline $\begin{array}{c}\text { R. rosea, without } \\
\text { test }\end{array}$ & $337 \pm 60$ * & $3163 \pm 142 *$ & $3.6 \pm 0.2 *$ & $203 \pm 48 *$ & $9.2 \pm 1.1 *$ & $1.8 \pm 0.1$ * & $11.4 \pm 0.7 *$ & $23.5 \pm 1.2 *$ \\
\hline R. rosea, after test & $173 \pm 36^{*}$ & $1986 \pm 107 *$ & $4.3 \pm 0.3 *$ & $706 \pm 204^{*}$ & $3.7 \pm 0.7^{*}$ & $2.3 \pm 0.2 *$ & $10.2 \pm 0.8^{*}$ & $12.0 \pm 0.7$ * \\
\hline
\end{tabular}

It is worth noting that the oral administration of $R$. adamsii extract to intact mice, in the absence of physical activity, was not accompanied by pronounced changes in the analyzed parameters of carbohydrate and energy metabolism; these results meet the basic requirement of an adaptogenic drug, which should mainly work during periods of stress and provide minimal activity under normal conditions [2]. All of the foregoing indicates that $R$. adamsii leaf extract provides a positive therapeutic effect on animals during situations of strong physical stress, similar to the way known herbal adaptogens (Rhodiola rosea, Eleuterococcus senticosus, Schizandra chinensis) affect these animals [4].

\section{Conclusions}

In the situation of the global catastrophe caused by the COVID-19 epidemic, scientific findings relating to drugs that mobilize the internal reserves of protective barriers of the human body are highly relevant [85]. Adaptogenic plant drugs are considered as possible prophylactic agents for alleviating the severity of COVID-19 symptoms [86]. The expansion of the range of known plant adaptogens in this regard is an extremely important scientific mission. Thus, we can give the first detailed opinion concerning the veracity of early ethnopharmacological views of $R$. adamsii leaf extracts providing positive effects on humans as an adaptogenic remedy. Additional experiments will be necessary to understand the mechanisms of activity of R. adamsii extracts, as well as to elucidate their safety data. Nonetheless, it is now possible to say that Sagan Dalya tea has good prospects for medical and therapeutic use.

Supplementary Materials: The following are available online at https:/ /www.mdpi.com/article/10 .3390 /antiox10060863/s1, Table S1: Reference standards used for the qualitative and quantitative analysis by HPLC-DAD-ESI-tQ-MS and HPLC-UV assays, Table S2: Regression equations, correlation coefficients, standard deviation, limits of detection, limits of quantification and linear ranges for 55 reference standards, Table S3: Retention times, UV- and ESI-MS spectral data of compounds 1-171 found in Rhododendron adamsii, Table S4: UV-spectral patterns of compounds found in R. adamsii, Figure S1: High-performance anion-exchange chromatography with photodiode detection chromatograms of free carbohydrates of Rhododendron adamsii leaves and reference standards, Figure S2: High-performance liquid chromatography with photodiode detection chromatograms of triterpenic acids of $R$. adamsii leaves and reference standards, Figure S3: HPLC-PDA chromatograms of ether and methanolic extracts of fresh R. adamsii leaves, Figure S4: Yield of ether extract from R. adamsii leaves collected in various months. 
Author Contributions: Conceptualization, D.N.O.; methodology, D.N.O. and N.K.C.; software, D.N.O.; validation, N.K.C.; formal analysis, N.K.C. and V.M.N.; investigation, D.N.O., N.K.C., and V.M.N.; resources, D.N.O. and N.K.C.; data curation, V.M.N.; writing—original draft preparation, D.N.O.; writing-review and editing, D.N.O.; visualization, D.N.O.; supervision, N.K.C.; project administration, N.K.C.; funding acquisition, D.N.O., N.K.C., and V.M.N. All authors have read and agreed to the published version of the manuscript.

Funding: This research was funded by the Ministry of Education and Science of Russia, grant numbers FSRG-2020-0019, 121030100227-7, and the Russian Foundation of Basic Research, grant number 19-09-00361.

Institutional Review Board Statement: The study was approved by the Russian Health Ministry (protocol code 708H, 23 August 2010) and the Ethics Committee of Institute of General and Experimental Biology (protocol code LM-0324, 28 January 2014).

Informed Consent Statement: Not applicable.

Data Availability Statement: Data is contained within the article.

Acknowledgments: The authors acknowledge the Buryat Research Resource Center for the technical support in chromatographic and mass-spectrometric research and also the students of the NorthEastern Federal University (Yakutsk, Russia) for helping to collect plant samples. The authors also are very thankful to Denis Kochetkov (Amur Oblast, Russia) for kindly providing the photo of Rhododendron adamsii.

Conflicts of Interest: The authors declare no conflict of interest. The funders had no role in the design of the study; in the collection, analyses, or interpretation of data; in the writing of the manuscript, or in the decision to publish the results.

\section{References}

1. Panossian, A.G.; Efferth, T.; Shikov, A.N.; Pozharitskaya, O.N.; Kuchta, K.; Mukherjee, P.K.; Banerjee, S.; Heinrich, M.; Wu, W.; Guo, D.; et al. Evolution of the adaptogenic concept from traditional use to medical systems: Pharmacology of stress- and aging-related diseases. Med. Res. Rev. 2021, 41, 630-703. [CrossRef]

2. Panossian, A. Understanding adaptogenic activity: Specificity of the pharmacological action of adaptogens and other phytochemicals. Ann. N. Y. Acad. Sci. 2017, 1401, 49-64. [CrossRef]

3. Dimpfel, W.; Schombert, L.; Keplinger-Dimpfel, I.K.; Panossian, A. Effects of an adaptogenic extract on electrical activity of the brain in elderly subjects with mild cognitive impairment: A randomized, double-blind, placebo-controlled, two-armed cross-over study. Pharmaceuticals 2020, 13, 45. [CrossRef] [PubMed]

4. Panossian, A.; Wikman, G. Effects of adaptogens on the central nervous system and the molecular mechanisms associated with their stress-protective activity. Pharmaceuticals 2010, 3, 188-224. [CrossRef] [PubMed]

5. Malyschev, L.I. (Ed.) Flora of Siberia, Pyrolaceae-Lamiaceae; CRC Press: Boca-Raton, FL, USA, 2006; Volume 11, pp. 16-19.

6. Dugarzhapov, T.A.; Basaev, S.E. Myths and Legends of Buryats; Novaya Buryatia: Ulan-Ude, Russia, 2017 ; pp. 34-45.

7. Haldar, J.R. Early Buddhist Mythology; Manohar: New Delhi, India, 2019; pp. 115-120.

8. Batorova, S.M.; Yakovlev, G.P.; Aseeva, T.A. Reference-Book of Traditional Tibetan Medicine Herbs; Nauka: Novosibirsk, Russia, 2013; pp. 182-183.

9. Ivanov, B.I. (Ed.) Atlas of Medicinal Plants of Yakutia; YaNTc SO RAN: Yakutsk, Russia, 2005; pp. 128-129.

10. Belenovskaya, L.M.; Lesiovskaya, E.E. (Eds.) Plant Resources of Russia: Actinidiaceae—Malvaceae; KMK: Saint Petersburg, Russia, 2009; Volume 2, pp. 41-50.

11. Rogachev, A.D.; Fomenko, V.V.; Sal'nikova, O.I.; Pokrovskii, L.M.; Salakhutdinov, N.F. Comparative analysis of essential oil compositions from leaves and stems of Rhododendron adamsii, R. aureum, and R. dauricum. Chem. Nat. Comp. 2006, 42, 426-430. [CrossRef]

12. Komarova, N.I.; Rogachev, A.D.; Chernyak, E.I.; Morozov, S.V.; Fomenko, V.V.; Salakhutdinov, N.F. Quantitative HPLC determination of main flavonoid content of Rhododendron adamsii leaves and stems. Chem. Nat. Comp. 2009, 45, 27-31. [CrossRef]

13. Razgonova, M.; Zakharenko, A.; Ercisli, S.; Grudev, V.; Golokhvast, K. Comparative analysis of Far East Sikhotinsky rhododendron (Rh. sichotense) and East Siberian rhododendron (Rh. adamsii) using supercritical $\mathrm{CO}_{2}$-extraction and HPLC-ESI-MS/MS spectrometry. Molecules 2020, 25, 3774. [CrossRef] [PubMed]

14. Olennikov, D.N.; Dudareva, L.V.; Osipenko, S.N.; Penzina, T.A. Chemical composition of essential oils from leaves of Rhododendron dauricum and Rh. aureum. Chem. Nat. Comp. 2009, 45, 450-452. [CrossRef]

15. Olennikov, D.N.; Dudareva, L.V.; Osipenko, S.N.; Penzina, T.A. Chemical composition of Rhododendron aureum (gold rosebay) essential oil from Pribaikal'e (Russian Federation). J. Serb. Chem. Soc. 2010, 75, 209-215. [CrossRef]

16. Olennikov, D.N.; Tankhaeva, L.M. Phenolic compounds from Rhododendron dauricum from Baikal region. Chem. Nat. Comp. 2010, 46, 471-473. [CrossRef] 
17. Bradford, M.M. A rapid and sensitive method for the quantification of microgram quantities of protein utilizing the principle of protein-dye binding. Anal. Biochem. 1976, 76, 248-254. [CrossRef]

18. Olennikov, D.N.; Tankhaeva, L.M. Absorption spectra of carbohydrates and related compounds in $\mathrm{H}_{2} \mathrm{SO}_{4}$. Chem. Nat. Comp . 2006, 42, 262-264. [CrossRef]

19. Olennikov, D.N.; Tankhaeva, L.M.; Samuelsen, A.B. Quantitative analysis of polysaccharides from Plantago major leaves using the Dreywood method. Chem. Nat. Comp. 2006, 42, 265-268. [CrossRef]

20. Olennikov, D.N.; Tankhaeva, L.M. Quantitative determination of phenolic compounds in Mentha piperita leaves. Chem. Nat. Comp. 2010, 46, 22-27. [CrossRef]

21. Chirikova, N.K.; Olennikov, D.N.; Tankhaeva, L.M. Quantitative determination of flavonoid content in the aerial part of Baical scullcap (Scutellaria baicalensis Georgi). Russ. J. Bioorg. Chem. 2010, 36, 915-922. [CrossRef]

22. Damien Dorman, H.J.; Shikov, A.N.; Pozharitskaya, O.N.; Hiltunen, R. Antioxidant and pro-oxidant evaluation of a Potentilla alba L. rhizome extract. Chem. Biodiv. 2011, 8, 1344-1356. [CrossRef]

23. Sun, B.; Ricardo-da-Silva, J.M.; Spranger, I. Critical factors of vanillin assay for catechins and proanthocyanidins. J. Agric. Food Chem. 1998, 46, 4267-4274. [CrossRef]

24. Porter, L.J.; Hrstich, L.N.; Chan, B.G. The conversion of procyanidins and prodelphinidins to cyanidin and delphinidin. Phytochemistry 1986, 25, 223-230. [CrossRef]

25. Olennikov, D.N.; Chirikova, N.K.; Okhlopkova, Z.M.; Zulfugarov, I.S. Chemical composition and antioxidant activity of Tánara Ótó (Dracocephalum palmatum Stephan), a medicinal plant used by the North-Yakutian nomads. Molecules 2013, 18, 14105-14121. [CrossRef]

26. Olennikov, D.N.; Kashchenko, N.I.; Chirikova, N.K.; Gornostai, T.G.; Selyutina, I.Y.; Zilfikarov, I.N. Effect of low temperature cultivation on the phytochemical profile and bioactivity of Arctic plants: A case of Dracocephalum palmatum. Int. J. Mol. Sci. 2017, 18, 2579. [CrossRef]

27. Mori, K.; Kidawara, M.; Iseki, M.; Umegaki, C.; Kishi, T. A simple fluorometric determination of vitamin C. Chem. Pharm. Bull. 1998, 46, 1474-1476. [CrossRef] [PubMed]

28. Olennikov, D.N.; Chirikova, N.K.; Vasilieva, A.G.; Fedorov, I.A. LC-MS profile, gastrointestinal and gut microbiota stability and antioxidant activity of Rhodiola rosea herb metabolites: A comparative study with subterranean organs. Antioxidants 2020, 9, 526. [CrossRef]

29. Olennikov, D.N.; Vasilieva, A.G.; Chirikova, N.K. Fragaria viridis fruit metabolites: Variation of LC-MS profile and antioxidant potential during ripening and storage. Pharmaceuticals 2020, 13, 262. [CrossRef]

30. Olennikov, D.N.; Tankhaeva, L.M.; Agafonova, S.V. Antioxidant components of Laetiporus sulphureus (Bull.: Fr.) Murr. fruit bodies. Appl. Biochem. Microbiol. 2011, 47, 419-425. [CrossRef]

31. Kumar, S.; Kumar, D.; Jusha, M.; Saroha, K.; Singif, N.; Vashishta, B. Antioxidant and free radical scavenging potential of Citrullus colocynthis (L.) Schrad. methanolic fruit extract. Acta Pharm. 2008, 58, 215-220. [CrossRef] [PubMed]

32. Olennikov, D.N.; Kashchenko, N.I.; Chirikova, N.K. A novel HPLC-assisted method for investigation of the Fe $\mathrm{Fe}^{2+}$-chelating activity of flavonoids and plant extracts. Molecules 2014, 19, 18296-18316. [CrossRef]

33. Olennikov, D.N.; Gadimli, A.I.; Isaev, J.I.; Kashchenko, N.I.; Prokopyev, A.S.; Katayeva, T.N.; Chirikova, N.K.; Vennos, C. Caucasian Gentiana species: Untargeted LC-MS metabolic profiling, antioxidant and digestive enzyme inhibiting activity of six plants. Metabolites 2019, 9, 271. [CrossRef]

34. Olennikov, D.N. Synanthropic plants as an underestimated source of bioactive phytochemicals: A case of Galeopsis bifida (Lamiaceae). Plants 2020, 9, 1555. [CrossRef]

35. Olennikov, D.N.; Chirikova, N.K.; Kashchenko, N.I.; Nikolaev, V.M.; Kim, S.-W.; Vennos, C. Bioactive phenolics of the genus Artemisia (Asteraceae): HPLC-DAD-ESI-TQ-MS/MS profile of the Siberian species and their inhibitory potential against $\alpha$-amylase and $\alpha$-glucosidase. Front. Pharmacol. 2018, 9, 756. [CrossRef]

36. Kedare, S.B.; Singh, R.P. Genesis and development of DPPH method of antioxidant assay. J. Food Sci. Technol. 2011, 48, 412-422. [CrossRef]

37. Malkoç, M.; Laghari, A.Q.; Kolayli, S.; Can, Z. Phenolic composition and antioxidant properties of Rhododendron ponticum: Traditional nectar source for mad honey. Anal. Chem. Lett. 2016, 6, 224-231. [CrossRef]

38. Lin, C.-Y.; Lin, L.-C.; Ho, S.-T.; Tung, Y.-T.; Tseng, Y.-H.; Wu, J.-H. Antioxidant activities and phytochemicals of leaf extracts from 10 native Rhododendron species in Taiwan. Evid. Based Complement. Altern. Med. 2014, 2014, 283938. [CrossRef]

39. Jing, L.; Ma, H.; Fan, P. Antioxidant potential, total phenolic and total flavonoid contents of Rhododendron anthopogonoides and its protective effect on hypoxia-induced injury in PC12 cells. BMC Complement. Altern. Med. 2015, 15, 287. [CrossRef] [PubMed]

40. Rafi, M.; Febriany, S.; Wulandari, P.; Suparto, I.H.; Ridwan, T.; Rahayu, S.; Siswoyo, D.M. Total phenolics, flavonoids, and anthocyanin contents of six Vireya rhododendron from Indonesia and evaluation of their antioxidant activities. J. Appl. Pharm. Sci. 2018, 8, 49-54. [CrossRef]

41. Dai, L.; He, J.; Miao, X.; Guo, X.; Shang, X.; Wang, W.; Li, B.; Wang, Y.; Pan, H.; Zhang, J. Multiple biological activities of Rhododendron przewalskii Maxim. extracts and UPLC-ESI-Q-TOF/MS characterization of their phytochemical composition. Front. Pharmacol. 2021, 12, 599778. [CrossRef] [PubMed]

42. Chen, T.-S.; Liou, S.-Y.; Chang, Y.-L. Antioxidant evaluation of three adaptogen extracts. Am. J. Chin. Med. 2008, 36, 1209-1217. [CrossRef] 
43. Sanjay, K.; Ameya, K.; Mauro, B.; Andrea, M. Antioxidant, anti-inflammatory, and adaptogenic activity of Asparagus acutifolius extract. Oriental Pharm. Exp. Med. 2009, 9, 83-89. [CrossRef]

44. Pal Singh, I.; Bharate, S.B. Phloroglucinol compounds of natural origin. Nat. Prod. Rep. 2006, 23, 558-591. [CrossRef]

45. Xu, W.-H.; Liang, Q.; Zhang, Y.-J.; Zhao, P. Naturally occurring arbutin derivatives and their bioactivities. Chem. Biodiv. 2015, 12, 54-81. [CrossRef]

46. Macbeth, A.K.; Mackay, J. LXXXIV. Studies of the glucosides. Part II. Arbutin. J. Chem. Soc. Trans. 1923, 123, 717-724. [CrossRef]

47. Iwata, N.; Wang, N.; Yao, X.; Kitanaka, S. Structures and histamine release inhibitory effects of prenylated orcinol derivatives from Rhododendron dauricum. J. Nat. Prod. 2004, 67, 1106-1109. [CrossRef]

48. Olennikov, D.N. Makisterone C-20,22-acetonide from Rhaponticum uniflorum. Chem. Nat. Comp. 2018, 54, 930-933. [CrossRef]

49. Qiang, Y.; Zhou, B.; Gao, K. Chemical constituents of plants from the genus Rhododendron. Chem. Biodiv. 2011, 8, 792-815. [CrossRef] [PubMed]

50. Ismail, N.; Alam, M. A novel cytotoxic flavonoid glycoside from Physalis angulata. Phytochemistry 2001, 72, 676-679. [CrossRef]

51. Parker, W.H.; Bohm, B.A. Flavonol glycosides of Limnanthes douglasii. Phytochemistry 1975, 14, 553-555. [CrossRef]

52. Louis, A.; Petereit, F.; Lechtenberg, M.; Deters, A.; Hensel, A. Phytochemical characterization of Rhododendron ferrugineum and in vitro assessment of an aqueous extract on cell toxicity. Planta Med. 2010, 76, 1550-1557. [CrossRef] [PubMed]

53. Heinke, R.; Arnold, N.; Wessjohann, L.; Schmidt, J. Negative ion tandem mass spectrometry of prenylated fungal metabolites and their derivatives. Anal. Bioanal. Chem. 2013, 405, 177-189. [CrossRef]

54. Iijima, M.; Munakata, R.; Takahashi, H.; Kenmoku, H.; Nakagawa, R.; Kodama, T.; Asakawa, Y.; Abe, I.; Yazaki, K.; Kurosaki, F.; et al. Identification and characterization of daurichromenic acid synthase active in anti-HIV biosynthesis. Plant Physiol. 2017, 174, 2213-2230. [CrossRef] [PubMed]

55. Clifford, M.N.; Knight, S.; Kuhnert, N. Discriminating between the six isomers of dicaffeoylquinic acids by LC-MS ${ }^{\mathrm{n}}$. J. Agric. Food Chem. 2005, 53, 3821-3832. [CrossRef]

56. Olennikov, D.N. Free carbohydrates, glucofructans, and other polysaccharides from Rhaponticum uniflorum. Chem. Nat. Comp. 2018, 54, 751-754. [CrossRef]

57. Fan, C.Q.; Yang, G.J.; Zhao, W.M.; Ding, B.Y.; Qin, G.W. Phenolic components from Rhododendron latoucheae. Chin. Chem. Lett. 1999, 10, 567-570.

58. Rawat, P.; Rai, N.; Kumar, N.; Bachheti, R.K. Review on Rhododendron arboreum-A magical tree. Orient. Pharm. Exp. Med. 2017, 17, 297-308. [CrossRef]

59. Belova, N.V.; Fokina, G.A. The triterpenoids of some species of Rhododendron. Chem. Nat. Comp. 1970, 6, 134. [CrossRef]

60. Fokina, G.A. Triterpene acids of Rhododendron plants of the flora of the USSR. Chem. Nat. Comp. 1979, 15, 651-652. [CrossRef]

61. Bohm, B.A. Intraspecific flavonoid variation. Bot. Rev. 1987, 53, 197-279. [CrossRef]

62. Gülck, T.; Møller, B.L. Phytocannabinoids: Origins and biosynthesis. Trends Plant Sci. 2020, 25, 985-1004. [CrossRef]

63. Mechoulam, R. Chemistry of Cannabis. In Psychotropic Agents. Handbook of Experimental Pharmacology; Hoffmeister, F., Stille, G., Eds.; Springer: Berlin/Heidelberg, Germany, 1982; Volume 55. [CrossRef]

64. Taura, F.; Iijima, M.; Kurosaki, F. Daurichromenic acid and grifolic acid: Phytotoxic meroterpenoids that induce cell death in cell culture of their producer Rhododendron dauricum. Plant Sign. Behav. 2018, 13, e1422463. [CrossRef] [PubMed]

65. Deepak, H.V.; Swamy, M.M.M.; Murai, Y.; Suga, Y.; Anetai, M.; Yo, T.; Kuragano, M.; Uwai, K.; Tokuraku, K.; Monde, K. Daurichromenic acid from the Chinese traditional medicinal plant Rhododendron dauricum inhibits sphingomyelin synthase and A $\beta$ aggregation. Molecules 2020, 25, 4077. [CrossRef] [PubMed]

66. Shishkin, B.K.; Bobrov, E.G. (Eds.) Flora USSR; AN SSSR: Moscow, Russia, 1952; Volume 18, pp. $43-47$.

67. Joshi, Y.C.; Dobhal, M.P.; Joshi, B.C.; Barar, F.S.K. Chemical investigation and biological screening of the stem of Rhododendron anthopogon (D. Don.). Pharmazie 1981, 36, 381.

68. Sharma, N.; Sharma, U.K.; Gupta, A.P.; Sinha, A.K. Simultaneous determination of epicatechin, syringic acid, quercetin-3-Ogalactoside and quercitrin in the leaves of Rhododendron species by using a validated HPTLC method. J. Food Comp. Anal. 2010, 23, 214-219. [CrossRef]

69. Dai, S.-J.; Chen, R.-Y.; Yu, D.-Q. Studies on the flavonoid compounds of Rhododendron anthopogonoides. Zhongguo Zhongyao Zazhi 2004, 29, 47.

70. Fan, M.-X.; Zhao, J.-Q.; Yuan, X.; Tao, Y.-D.; Shao, Y.; Mei, L.-J. Chemical constituents from Rhododendron anthopogonoides. Chin. Trad. Herb. Drugs 2010, 47, 3769-3772. [CrossRef]

71. Iwata, N.; Kitanaka, S. Tetracyclic chromane derivatives from Rhododendron anthopogonoides. J. Nat. Prod. 2010, 73, 1203-1206. [CrossRef] [PubMed]

72. Iwata, N.; Kitanaka, S. New cannabinoid-like chromane and chromene derivatives from Rhododendron anthopogonoides. Chem. Pharm. Bull. 2011, 59, 1409-1412. [CrossRef] [PubMed]

73. Ahmad, S.; Mir, I.; Hussain, G.; Galbraith, M.N. Noreugenin from Rhododendron collettianum. Phytochemistry 1973, 12, 727-728. [CrossRef]

74. Hakeem Said, I.; Rezk, A.; Hussain, I.; Grimbs, A.; Shrestha, A.; Schepker, H.; Brix, K.; Ullrich, M.S.; Kuhnert, N. Metabolome comparison of bioactive and inactive Rhododendron extracts and identification of an antibacterial cannabinoid(s) from Rhododendron collettianum. Phytochem. Anal. 2017, 28, 454-464. [CrossRef] 
75. Modak, B.; Torres, R.; Urzúa, A. Seasonal variation of the flavonoids pinocembrin and 3-O-methylgalangin, in the surface component mixture (resinous exudates and waxy coating) of Heliotropium stenophyllum. J. Chil. Chem. Soc. 2011, 56, 532-534. [CrossRef]

76. Fischbach, R.; Kossmann, B.; Panten, H.; Steinbrecher, R.; Heller, W.; Seidlitz, H.; Sandermann, H.; Hertkorn, N.; Schnitzler, J. Seasonal accumulation of ultraviolet-B screening pigments in needles of Norway spruce (Picea abies (L.) Karst.). Plant Cell Environ. 1999, 22, 27-37. [CrossRef]

77. Bouzoubaâ, Z.; El Mousadik, A.; Belahsen, Y. Variation in amounts of epicuticular wax on leaves of Argania spinosa (L). Skeels. Acta Bot. Gallica 2006, 153, 167-177. [CrossRef]

78. Zabkiewicz, J.A.; Gaskin, R.E. Seasonal variation of gorse (Ulex europaeus L) surface wax and trichomes. New Phytol. 1978, 81, 367-373. [CrossRef]

79. Kwak, M.J.; Lee, J.K.; Park, S.; Kim, H.; Lim, Y.J.; Lee, K.-A.; Son, J.-a; Oh, C.-Y.; Kim, I.; Woo, S.Y. Surface-based analysis of leaf microstructures for adsorbing and retaining capability of airborne particulate matter in ten woody species. Forests 2020, 11, 946. [CrossRef]

80. Gulcin, İ. Antioxidants and antioxidant methods: An updated overview. Arch. Toxicol. 2020, 94, 651-715. [CrossRef] [PubMed]

81. Agati, G.; Brunetti, C.; Fini, A.; Gori, A.; Guidi, L.; Landi, M.; Sebastiani, F.; Tattini, M. Are flavonoids effective antioxidants in plants? Twenty years of our investigation. Antioxidants 2020, 9, 1098. [CrossRef]

82. Song, J.; Manir, M.M.; Moon, S.-S. Cytotoxic grifolin derivatives isolated from the wild mushroom Boletus pseudocalopus (Basidiomycetes). Chem. Biodiv. 2009, 6, 1435-1442. [CrossRef] [PubMed]

83. Shikov, A.N.; Lazukina, M.A.; Pozharitskaya, O.N.; Makarova, M.N.; Golubeva, O.V.; Makarov, V.G.; Djachuk, G.I. Pharmacological evaluation of Potentilla alba L. in mice: Adaptogenic and central nervous system effects. Pharm. Biol. 2011, 49, 1023-1028. [CrossRef] [PubMed]

84. Domene, A.M. Effects of adaptogen supplementation on sport performance. A recent review of published studies. J. Hum. Sport Exerc. 2013, 8, 1054-1066. [CrossRef]

85. Mahmood, N.; Nasir, S.B.; Hefferon, K. Plant-based drugs and vaccines for COVID-19. Vaccines 2021, 9, 15. [CrossRef] [PubMed]

86. Panossian, A.; Brendler, T. The role of adaptogens in prophylaxis and treatment of viral respiratory infections. Pharmaceuticals 2020, 13, 236. [CrossRef] [PubMed] 